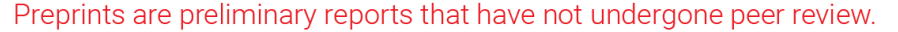 \\ They should not be considered conclusive, used to inform clinical practice, or referenced by the media as validated information. \\ Expanded and Filtered Features based ELM Model for Thyroid Disease Classification
}

Kapil Juneja ( $\sim$ kapil.juneja81@gmail.com )

Maharshi Dayanand University Rohtak https://orcid.org/0000-0002-6351-3351

\section{Research Article}

Keywords: Thyroid, Machine Learning, Classification, Feature Selection, Prediction, ELM

Posted Date: April 14th, 2021

DOl: https://doi.org/10.21203/rs.3.rs-385005/v1

License: (c) (i) This work is licensed under a Creative Commons Attribution 4.0 International License. Read Full License 


\section{Title Page}

Title

Authors

Corresponding Author :

Affiliation

Permanent Address

Present Address

Mobile Number

Email ID

ORC ID

Scopus ID

\section{Expanded and Filtered Features based ELM Model for Thyroid Disease Classification}

Kapil Juneja

Kapil Juneja

Maharshi Dayanand University, Rohtak, Haryana, India

307, Sector 14

Rothak (124001)

Haryana, India

307, Sector 14

Rothak (124001)

Haryana, India

+91-9896654464

kapil.juneja81@gmail.com

0000-0002-6351-3351

57188582145 


\title{
Expanded and Filtered Features based ELM Model for Thyroid Disease Classification
}

\begin{abstract}
Thyroid disorder affects the regulation of various metabolic processes throughout the human body. The structural and functional disorders can affect the body as well as the brain. The computer-aided diagnosis system can identify the kind of Thyroid disease. One such machine learning framework is presented in this paper to recognize disease existence and type. In this paper, a fuzzy adaptive feature filtration, expansion, and again filtration based model is presented for generating the most relevant and contributing features. This two-level filtration model is processed in a controlled fuzzy-based multimeasure evaluation. At the first level, the composite-fuzzy measures are combined with expert's recommendations for identifying the ranked and relevant features. At the second level, the statistical computation based distance measure is applied for expanding the featureset. The fuzzification is applied to expanded featureset for transiting the continuous values to fuzzy-values. At this level, the fuzzy-based composite-measure is applied for selecting the most contributing and relevant features over the expanded dataset. This processing featureset is processed by the ELM classifier to predict the disease existence and class. Five experiments are conducted on two datasets for validating the performance and reliability of the proposed framework. The comparative analysis is conducted against the NaiveBayes, Decision Tree, Decision Forest, Random Tree, Multilevel Perceptron, and RBF Networks. The analysis outcome is taken in terms of accuracy, error, and relevancy based parameters. The proposed framework clams the significant gain in accuracy, relevancy, and reduction in the error rate.
\end{abstract}

Keywords: Thyroid; Machine Learning; Classification; Feature Selection; Prediction; ELM

\section{Introduction}

Thyroid[1,2,3] is one of the common endocrine disorder which is spreading worldwide. Even in India, more than 50 million people are suffering from thyroid disease. The thyroid is a butterfly-shaped gland located in the neck. It controls the metabolism in the human body. It means, it can affect the complete functioning associated with the hormones. Thyroid disorder[2,3,4,5,6] can be identified easily as the small swelling in the throat, and the accessibility of its diagnosis and treatment is 
easy. The thyroid gland has mainly two types of hormones called Triiodothyronine (T3) and Thyroxine (T4). These hormones control the body temperature, protein production, energy production, and circulation. Iodine is the main structural substance of these two hormones. The imbalance of these hormones can cause a different kinds of thyroid disorders. There are four different forms of thyroid disorder

\subsection{Hypothyroidism}

When the thyroid gland does not produce enough hormones, it is called low thyroid or underactive thyroid or Hypothyroidism. It can cause depression, constipation, heavyweight, cold, etc. The occasional swelling is also the symptom of Hypothyroidism.

\subsection{Hyperthyroidism}

When the thyroid gland produces the hormones in access, such a situation is called excessive thyroid or Hyperthyroidism. It can be identified as muscle weakness, heat intolerance, thyroid enlargement, faster heartbeat, sleeping problem, weight loss, etc.

\subsection{Structural Abnormalities}

The structural change or abnormality in the gland can also cause thyroid disease. Because of this abnormality, nodules can appear on the gland. This abnormal enlargement is called Goiter, and it can be removed by using a surgery of the organ.

\subsection{Tumors}

Another serious form of thyroid disorder is thyroid cancer. The cells that cause thyroid cancer are distinct from all other cells of the human body. These cells absorb iodine from the bloodstream, which is poisonous to the human body. This kind of disease can result in hair loss, body pain, sickness, nausea, etc. These surgical treatments are required to cure thyroid cancer.

The automated disorder identification system can be developed after recording the thyroid disease symptoms in terms of different hemoglobin contents, patient information, sonography based information, and structural information of Goiter. Various classification methods $[7,8,9,10,11]$ such as Bayesian network, neural network[12], SVM, etc. were used by the researchers in recent years. For reducing the computational complexity, the feature ranking and selection methods were provided by the researchers to optimize the classification accuracy. In this paper, a fuzzadaptive composite-measure is applied0 at two-levels for reducing the dimension size and improving the relevancy of featureset. The featureset expansion is achieved using statistical-distance based computation. The expanded-filtered featureset is transited through fuzzy rules for handling the ambiguities. The final-relevant featureset is processed under the ELM classifier for predicting the thyroid existence and disease class. 


\subsection{Major Contributions}

The problem in the thyroid gland can cause various health issues, including fatigue, weakness, hair loss, blood pressure, etc. The overactive and underactive thyroid gland is having a different impact on health. It is necessary to diagnose the thyroid accurately with category specification. In this research, a selective and extensive feature processed model is presented for accurate diagnose of the thyroid. The contribution of this research is listed hereunder:

- A Fuzzy adaptive composite-measure and recommendation analysis based feature selection method is included for the identification of relevant and contributing features.

- Fuzzy based Two-level filtration method is employed for generating an effective and reliable featureset.

- The feature expansion is conducted using statistical distance measures

- The impurities of continuous data are removed by applying fuzzy rules and generating fuzzy nominal data.

- The dynamic weight adjustment based ELM classifier is applied for accurate prediction of disease and disease class.

- Five different experiments are conducted for validating the performance of the proposed framework and achieved effective prediction results for $2,4,6$, and 18 class datasets.

In this paper, a filtered and expanded feature-based probabilistic model is presented to classify the thyroid disease. Fuzzy adaptive composite-measure based ranking and filtration is accomplished for identifying the most significant features. This filtration stage is applied at two-levels: before and after feature-expansion. The statistical aggregative measure is used for feature expansion, and the fuzzy rules are applied to validate the dataset. Finally, the dynamic weight adjustment based ELM model is employed to recognize the disease class. In this section, the detailed exploration of thyroid disease, its types, symptoms, and diagnosis is provided. The section also explored the feature selection and classification methods. In section II, the methods used by the researchers to classify the thyroid disease over different datasets are provided. In section III, the proposed expanded and filtered probabilistic model for thyroid disease classification is presented. The algorithmic behavior of this model is also provided. Detailed exploration of proposed fuzzy-based feature selection and expansion is also provided in this section. In section IV, the experimental results obtained from work on five experiments taken on two different datasets are discussed. In section $\mathrm{V}$, the conclusion and future scope of this work are provided.

\section{Related Work}

The patients of thyroid disease are increasing rapidly and suffering from various disease forms, including hyperthyroid, Hypothyroidism, thyroid nodule, cancer, and 
so on. Various data mining and machine learning[13, $\underline{14}, \underline{15}, \underline{16}, \underline{17}]$ algorithms were analyzed by the researchers for identifying the most significant, accurate and reliable method. The automated disease classification under the machine learning algorithm[18] is designed by the researchers to diagnose the disease based on the symptoms. Each stage of the machine learning algorithm was improved by the researchers to improve the accuracy of disease recognition. Pan et al.[2] used the PCA and random forest-based method for thyroid classification. The PCA is applied to preserve the variability, and the combined ensemble learning method improved the accuracy effectively. A comparative evaluation of various machine learning algorithms for thyroid classification was provided by Maysanjaya et al.[3]. The author applied the RBF (Radial Based Function), LVQ (Learning Vector Quantization), Multilayer Perceptron (MLP), Back Propagation Algorithm (BPA), Artificial Immune Recognition System (AIRS) and Perceptron classifiers. The analytical results provided by the author identified that the MLP provided a maximum accuracy of $96.74 \%$. A hybrid intelligent framework[19] was proposed by integrating the recursive feature elimination (RFE) with the SVM classifier. The teaching learningbased algorithm (TLBO) and Differential Evolution (DE) algorithms were integrated within the framework for optimizing the parameters of SVM. The average accuracy received by this model was $97.17 \%$. A Multi-layer perceptron based intelligent[20] method was investigated for effective recognition of thyroid patients. The model was implemented on a primary dataset collected for a hospital. The author collected 11 attributes and achieved 99.8\% accuracy. Yadav et al.[21] used the Decision Tree Ensemble approach for thyroid disease prediction. The method was implemented in the real environment on a real-time dataset and achieved $99.2 \%$ accuracy. An ensemble[22] approach of thyroid disease classification was defined by combining C4.5 and Random Forest techniques. The proposed method achieved 96\% accuracy on average.

The feature selection and ranking methods were opted by the researchers to process only the relevant features. Quereshi et al.[5] used the feature rejection technique to discard redundant and irrelevant features. The reduced dimensions featureset was processed under SVM, KNN, Neural Network, and Decision Tree methods to gain a higher classification rate. The ranker search[23] method was applied to identify the most relevant features from the thyroid disease dataset. The highest-ranked features were processed by the probabilistic Naive Bayes classifier to gain accuracy over $95 \%$. A fuzzy rule[24] based expert system was designed to process the most relevant features. K-Means and neuro-fuzzy-based model was designed to handle suspicious and uncertain conditions to build final rules. Huang et al.[25] compared four different feature selection methods called Chi-Square, Fisher Score, Information gain, and minimal-redundancy-maximum-relevance (mRMR) methods on a sub-health dataset. The accuracy-based verification was also provided on all feature selection methods using the KNN classifier. Another empirical evaluation of Binary Relevance, Pairwise, and Label power-set feature selection methods were provided by Rodriguez et al.[26]. Padmaja et al.[27] also provided an analytical and comparative measure for several feature selection methods, including Sequential Forward Selection (SFS), Sequential Floating Forward Selection (SFFS) and Random Subset Feature Selection (RSFS), etc. 
Prasad et al.[28] proposed the string matching algorithm and the bee colony optimization based hybrid approach for accurate prediction of thyroid disease. A regular match using a string matching algorithm achieved the $100 \%$ accuracy. But, when the matching failed, the method is replaced by PSO (Particle Swarm Optimization) method, which achieved the 93\% accuracy. Even if PSO failed, the higher method is replaced by ABC (Artificial Bee Colony) optimization method, which achieved the $65 \%$ accuracy. It shows, no single method has achieved effective accuracy. On average, $86 \%$ accuracy is achieved by the system irrespective of the failure ratio of each method. A rule-based classifier was designed by Yeh et al.[29] as an improvement to SSO (Simplified Swarm Optimization) for improving the solution quality of thyroid prediction. The rule is applied to feature pruning and to avoid the over-fitting of the training dataset. The computational results verified the superiority of the method over conventional and (Soft Computing) SC-based classifiers. A GDA_WSVM (Generalized Discriminant Analysis and Wavelet Support Vector Machine)[30] system was introduced for improving the accuracy of thyroid prediction. The method used the GDA (Generalized Discriminant Analysis) method as an extensive feature extraction and reduction approach for the correct diagnosis of thyroid disease. The author achieved a maximum accuracy of $91.86 \%$. A recursiverule[31] extraction based continuous attribute processing was defined for accurate and interpretable classification of thyroid disease. The author processed the imbalanced dataset and handled the impurities that exist in the dataset. This interpretable rulesbased method has achieved a maximum accuracy of $96.70 \%$. The accuracy of the method is controlled by the generated discrete and continuous rules. Better rule formation can be done to improve the accuracy of thyroid prediction.

Temurtas et al.[32] did a detailed study of thyroid disease diagnosis using neural networks. Different network configurations were analyzed by the author with different learning vectors and kernels. The minimum and maximum classification accuracy achieved by the author was $79.08 \%$ and $94.81 \%$. Kodaz et al.[33] used the information gain for feature filtration and applied the AIRS (Artificial immune recognition system) for thyroid diagnosis. The method achieved $95.90 \%$ accuracy. A fuzzy weighted[34] pre-processing based AIRS (Artificial Immune Recognition System) was proposed as a hybrid method for solving the diagnosis problem of thyroid disease. With sampling variations, $85 \%$ of accuracy was achieved by the author. Another hybrid immune-based thyroid disease classification was provided by Chang et al.[35]. The method was defined as an extension to GA (Genetic Algorithms). The probability analysis was applied to handle the diversity that exists in the data and to reduce the risk. The average accuracy achieved by the method was 96.78\%. The hybridization of LDA (Linear Discriminant Analysis), Kth nearestNeighbor (KNN), and ANFIS (Adaptive Neurofuzzy Interference System) were provided by Ahmad et al.[36] for effective thyroid disease forecasting. LDA was applied by the author for feature extraction, and then filtration was performed using the weighted-KNN approach. The method achieved a higher accuracy of $98.5 \%$. The comparative evaluation of conventional classifiers was provided by Pal et al.[37] for thyroid detection. The author identified the KNN as the best performing classifier with $96.90 \%$ accuracy over Naive Bayes and SVM classifiers.

An integrated model using SOM (Self Organizing Map) and LVQ (Linear Vector Quantization) was proposed by Razia et al.[7] for identification of thyroid disease 
class. This integrated model achieved 85\% accuracy. Shankar et al.[38] improved the multi-kernel SVM approach for thyroid disease classification by integrating a feature selection approach at an intermediate layer. The feature selection method identified the insignificant features and computationally improved the performance of the model. This filtered featureset based method has achieved $97.49 \%$ accuracy.

Radiologists and physicians also use sonography images to diagnose the thyroid nodules. The textural features[4] were extracted and processed in SVM (Support Vector Machine) to classify thyroid lesions. The author processed the 78 textural features and validated them using the 3-fold method to gain accuracy over $98 \%$.

\section{Research Methodology}

The thyroid is a hormone generated disease which can be identified by analyzing the symptoms and medical history of patients. The thyroid gland produces various kinds of hormones that affect every cell, organs, and tissues to regulate the metabolism of the human body. The intensity of thyroid hormone affects the body's energy, temperature, and production-proteins. Various symptoms, body variations, and tests are analyzed by the experts to diagnose the type of thyroid. In this paper, an effective machine learning model is presented that observes and processes the available symptoms and test-measures to identify the type of thyroid infection accurately. In this paper, a fuzzy adaptive feature selection and expansion method are provided for generating the most reliable and relevant features for thyroid prediction. The composite measures and recommendations were combined through fuzzy rules for generating the compact-efficient featureset. Later on, the dynamic weight adjustment based ELM classifier is applied for accurate prediction of thyroid existence and type. The proposed feature filtration and expansion based probabilistic model is provided in Figure 1.

The proposed framework has accepted the larger training set taken from the secondary [39] source. This raw training set is containing the patient information, disease symptoms, and various test results conducted on the patient. In the earlier phase of this proposed model, the evaluation of the raw training set is performed to identify the most contributing features. The feature selection is made using a fuzzy and composite measure based method. The composite measure defined in this work used the InfoGain, GainRatio, and ChiSq measures. The expert's recommendation is also combined to generate the fuzzy rules for generating the Level-I filtered dataset. The expert opinions are collected personally from the physician and online $[\underline{40}, \underline{41}]$ sources. The collective computation is applied to these fuzzy qualified measures and manual assessment weights to identify the most contributing features. At level II, the feature expansion is done by applying the statistical-distance measure. The statistical measures included in this work are Mean, Standard Deviation (SD), and Standard Error (SE). The fuzzy rules are applied to this expanded dataset for transiting the continuous features to fuzzy-nominal features. This expanded dataset is further processed by the fuzzy-based composite-measures for identifying the most contributing and relevant features. At the final stage, a dynamic weight adjustment based ELM classifier is applied for prediction of thyroid disease existence and type. 
The input-testing set or the new patient physiological, symptoms, and various test information is supplied to this proposed ML model to diagnose the type of thyroid disease. In this section, each of the integrated functionality of this proposed framework is provided.

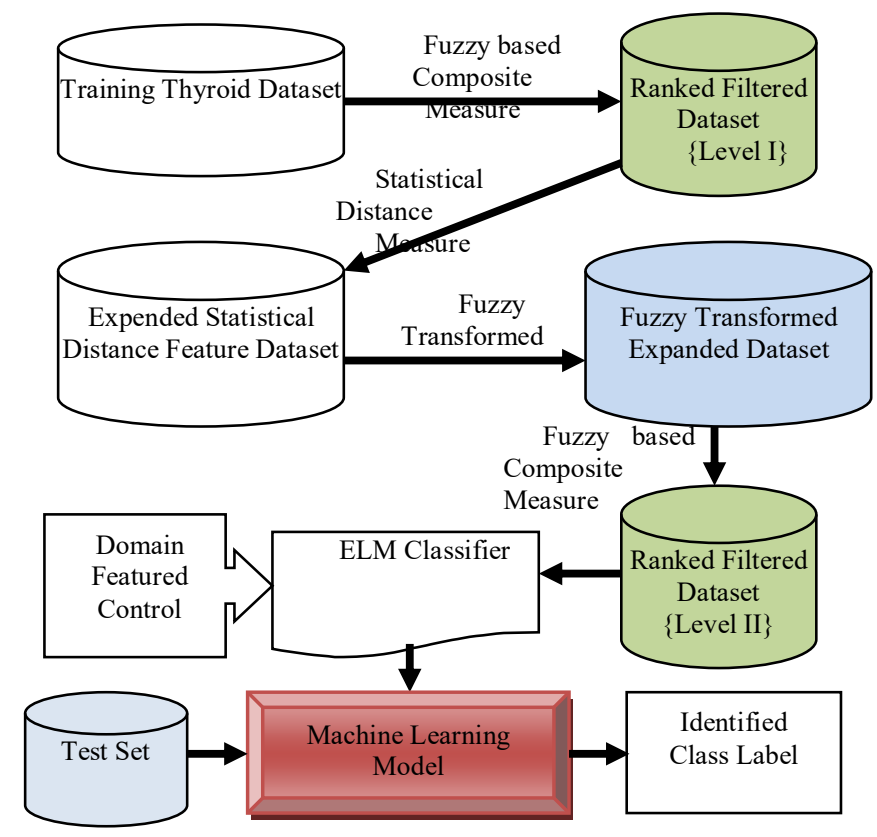

Figure 1: Fuzzy Filtered-Expended-Filtered Featured based ELM Model

\subsection{Effective and Ranked Thyroid Feature Selection (Level I)}

This research is formulated to diagnose the existence and category of thyroid disease in a patient. The wider real-time datasets are collected from the UCI repository. The description of datasets $[\underline{39}, \underline{42}]$ is provided in Section 4 . In the broader form, the dataset has 29 features for an individual. These features include physiological features, Patient Information, Symptoms, and various test measures. Some of these features are basic features, including Age, Gender, and query information. The query information collects the case-history of the patient respective to earlier infections to thyroxine, antithyroid medication, hypothyroid, and hyperthyroid. The clinical information is collected in the form of low level and highlevel symptoms. The high-level symptoms include sickness, pregnancy, and physiological changes identified in the patient. The low-level symptoms include the size of Goitre, Infection of I131, and lithium. By consulting the physician (expert) personally and by taking the depth study of disease [40,41], it is identified that the physiological and general patient information are having a negligible contribution to diagnose the disease. The high level and low level clinical and query features with respective effective and doctor recommendations are provided in table 1 . The table identified that the infection of I131 and Lithium highly influences the thyroid 
hormones. These recommendations are considered as the weighted factor and used with feature measures to generate the ranked filtered featureset.

Table 1: Effect of Symptoms and Features of Thyroid Patients

\begin{tabular}{|c|c|c|}
\hline Features & Effect/Description & $\begin{array}{c}\text { Doctor } \\
\text { Recommendation }\end{array}$ \\
\hline $\mathrm{I} 131$ & Destroy Thyroid Cells & High \\
\hline $\begin{array}{l}\text { thyroid } \\
\text { surgery }\end{array}$ & $\begin{array}{c}\text { Complication Risks are less than } 2 \\
\text { percent }\end{array}$ & Low \\
\hline Pregnancy & $\begin{array}{l}\text { Changes exist in hormones and size } \\
\text { during pregnancy }\end{array}$ & Low \\
\hline Goiter & $\begin{array}{l}\text { Abnormal enlargement of the } \\
\text { thyroid gland }\end{array}$ & Medium \\
\hline Tumor & $\begin{array}{l}\text { Less occurred cancer, In later age } \\
\text { of an individual, Lesser visible } \\
\text { symptoms } \\
\end{array}$ & Low \\
\hline $\begin{array}{c}\text { Hypopituitar } \\
\text { y }\end{array}$ & $\begin{array}{c}\text { Rare Disorder, Affect blood } \\
\text { pressure, growth, etc. Results } \\
\text { hypopituitarism }\end{array}$ & Medium \\
\hline lithium & $\begin{array}{l}\text { A drug that affects the thyroid } \\
\text { function. It can develop Goiter, } \\
\text { Hypothyroidism and } \\
\text { Hyperthyroidism. }\end{array}$ & High \\
\hline Sick & $\begin{array}{l}\text { Sickness exists in thyroid problems, } \\
\text { but it is not a single reason. }\end{array}$ & Low \\
\hline psych & $\begin{array}{l}\text { Affects mentally, physically, and } \\
\text { emotionally. But difficult to identify it } \\
\text { is because of thyroid }\end{array}$ & Low \\
\hline
\end{tabular}

The physiological changes such as weakness, hair loss, blood pressure, etc. are not enough to diagnose thyroid disease. The blood tests such as T4, TSH, T3, FTI, and TBG are conducted by the expert to perform low-level evaluations. These tests are able to identify the degree of abnormality and the category of thyroid disease. The abnormality influence, type of disorder, associated-symptoms of these tests are provided in table 2. Based on the scope and recommendation of these tests, the weights are also assigned to these tests, which are listed in this table.

Table 2: Effect of Various Tests conducted for Thyroid Diagnosis

\begin{tabular}{|c|c|c|c|c|c|}
\hline $\begin{array}{l}\text { Test/Feat } \\
\text { ures }\end{array}$ & $\begin{array}{c}\text { Abnormal } \\
\text { High Results }\end{array}$ & $\begin{array}{l}\text { Abnormality } \\
\text { Low Results }\end{array}$ & Disorders & Symptoms & $\begin{array}{c}\text { Doctor } \\
\text { Recomm } \\
\text { endation }\end{array}$ \\
\hline TT4 Test & $\begin{array}{l}\text { High Protein } \\
\text { Level, } \\
\text { High Iodian } \\
\text { Pregnancy- } \\
\text { related } \\
\text { tumor }\end{array}$ & $\begin{array}{c}\text { Dietary } \\
\text { Issues } \\
\text { Protein } \\
\text { Level } \\
\text { hypothyroidi } \\
\text { sm } \\
\end{array}$ & $\begin{array}{l}\text { Hyperthyroi } \\
\text { dism, } \\
\text { Hypothyroid } \\
\text { ism, } \\
\text { Hypopituitar } \\
\text { ism }\end{array}$ & $\begin{array}{l}\text { Dryness, } \\
\text { Eye Issue, } \\
\text { Hair Loss, } \\
\text { Blood } \\
\text { pressure, } \\
\text { etc }\end{array}$ & High \\
\hline
\end{tabular}




\begin{tabular}{|c|c|c|c|c|c|}
\hline & $\begin{array}{l}\text { Germ cell } \\
\text { tumor }\end{array}$ & & & & \\
\hline T3 Test & $\begin{array}{c}\text { Graves } \\
\text { disease, } \\
\text { Painless } \\
\text { thyroidtis, } \\
\text { toxic } \\
\text { nodular } \\
\text { Goiter, } \\
\text { Periodic } \\
\text { paralysis, } \\
\text { Hyperthyroi } \\
\text { dism }\end{array}$ & $\begin{array}{l}\text { hypothyro } \\
\text { idism }\end{array}$ & $\begin{array}{l}\text { hyperthyroid } \\
\text { ism, } \\
\text { Hypopituitar } \\
\text { ism } \\
\text { Primary and } \\
\text { secondary } \\
\text { Hypothyroid } \\
\text { ism }\end{array}$ & $\begin{array}{c}\text { Weakness, } \\
\text { Fatigue, } \\
\text { Weight } \\
\text { loss, } \\
\text { sensitivity } \\
\text { increased, } \\
\text { Hair loss, } \\
\text { dryness, } \\
\text { etc. }\end{array}$ & Medium \\
\hline TSH & $\begin{array}{c}\text { More } \\
\text { Hormones } \\
\text { Overactive } \\
\text { thyroid } \\
\text { gland }\end{array}$ & $\begin{array}{l}\text { Underacti } \\
\text { ve thyroid } \\
\text { gland }\end{array}$ & $\begin{array}{l}\text { Hypothyroid } \\
\text { ism } \\
\text { Hyperthyroi } \\
\text { dism }\end{array}$ & $\begin{array}{c}\text { Through } \\
\text { Blood test }\end{array}$ & High \\
\hline $\begin{array}{c}\text { FTI } \\
\text { (Free T4) }\end{array}$ & $\begin{array}{l}\text { High Protein } \\
\text { Level, } \\
\text { High Iodian } \\
\text { Pregnancy- } \\
\text { related } \\
\text { tumor } \\
\text { Germ cell } \\
\text { tumor }\end{array}$ & $\begin{array}{l}\text { Dietary } \\
\text { Issues } \\
\text { Protein } \\
\text { Level } \\
\text { hypothyro } \\
\text { idism }\end{array}$ & $\begin{array}{l}\text { Hypothyroid } \\
\text { ism } \\
\text { Hyperthyroi } \\
\text { dism }\end{array}$ & & High \\
\hline $\begin{array}{c}\text { TBG } \\
\text { (thyroxin } \\
\text { e-binding } \\
\text { globulin) }\end{array}$ & $\begin{array}{c}\text { High Results } \\
\text { can be } \\
\text { normal in } \\
\text { pregnancy } \\
\text { or Liver } \\
\text { Disease }\end{array}$ & $\begin{array}{l}\text { Low can be } \\
\text { due to } \\
\text { acromegaly, } \\
\text { Acute } \\
\text { Illness, } \\
\text { Hyperthyroi } \\
\text { dism. } \\
\text { malnutrition }\end{array}$ & $\begin{array}{l}\text { hyperthyroid } \\
\text { ism }\end{array}$ & $\begin{array}{c}\text { Dryness, } \\
\text { fatigue, } \\
\text { hair loss, } \\
\text { sensitivity } \\
\text { Increased, } \\
\text { etc }\end{array}$ & Low \\
\hline
\end{tabular}

Table 2 identifies the functional behavior and its scope based on high and low abnormality. These tests are capable of identifying different categories of thyroid disease. The values of these tests and their relation to thyroid disease categories are provided in table 3. This relation shows that TSH, T4, and FTI tests are capable of diagnosing the Hyperthyroidism and Hypothyroidism accurately. T3 test can diagnose only Hyperthyroidism.

Table 3: Test Capabilities for Thyroid Disease Category Identification

\begin{tabular}{|l|l|l|l|l|}
\hline & Normal & Hyperthyroidis & $\begin{array}{c}\text { Hypothyroidis } \\
\text { m Primary }\end{array}$ & $\begin{array}{c}\text { Hypothyroidis } \\
\text { m Secondary }\end{array}$ \\
\hline TSH & Normal & Low & High & Low \\
\hline T4 & Normal & High & Low & Low \\
\hline FTI & Normal & High & Low & Low \\
\hline
\end{tabular}


\begin{tabular}{l|l|l|l|l|} 
T3 & Normal & High & - & - \\
\hline
\end{tabular} After getting the expert opinion, recommendation, and concern, the available dataset features are analyzed respectively to labeled diseases. Various featureselection and ranking methods are available for the identification of contributing features. The feature selection measures improve the efficiency and reliability of classification methods. The label-driven analysis is performed by these ranking measures. In section 3.1, the functional processing and ranking behavior of these measures are provided hereunder. In this paper, a composite and fuzzy rule-based measure is applied to these measures for identification. In section 3.2, the proposed composite rule-based evaluation, measures, and functional behavior is provided. The performance analysis of the proposed composite-fuzzy feature-based model is provided against independent feature rankers.

\subsection{Feature-Ranking and Selection Measures}

\subsection{Info-Gain}

Info-gain is the ranking[25,26,27] method used to evaluate the feature based on information theory and its mapping to the relative class. The predictive integrated evaluation measure as information obtained from the training set is shown in Equation (1).

$$
\operatorname{Iv} \phi \mathrm{O}(\Sigma)=-\sum_{j=1}^{K} p\left(C_{j}\right) \log _{2} p\left(C_{j}\right)
$$

Where $\mathrm{K}$ is the number of classes of thyroid disease disorder, $\mathrm{C}$ is the set of classes, $\mathrm{p}\left(\mathrm{C}_{\mathrm{j}}\right)$ is the probabilistic derivation of a number of elements of the particular sample set map to class $\mathrm{C}_{\mathrm{j}}$

The evaluation is further extended by specifying the range interval. Let for a particular feature $f_{i}$, the range interval $r_{k}\left(f_{i}\right)$ is defined for the feature, the number of samples that map the range is given by $n_{i k}$. The information obtained for a sample $S_{i k}$ is given in Equation (2) for the feature $f_{i}$ and respective to the defined interval $r_{k}\left(f_{i}\right)$.

$$
\operatorname{Iv} \phi \mathrm{O}\left(S_{i k}\right)=\square-\sum_{j=1}^{K} p_{i k j} \log _{2} p_{i k j}
$$

From this available feature range, $M$ intervals are identified, and the integrated information map is obtained as $\mathrm{E}_{\mathrm{i}}$. The interval specific information map is given by

$$
\mathrm{E}_{\mathrm{\imath}}=\sum_{k=1}^{M} p_{i k} I\left(S_{i k}\right)
$$

In the formalized and integrated form, the information gain is obtained and represented by equation (4).

$$
\mathrm{I} \Gamma_{\mathfrak{\imath}}=\mathrm{I}(\Sigma)-\mathrm{E}_{\iota}
$$

The information gain can be applied to individual independent features to assign the ranking. The features with higher can be selected as the high-value feature. Info-gain values obtained for various features of the Thyroid and Hypothyroid dataset are provided in Section 4. The evaluated values by the measure based on the instances of both datasets are shown in Figure 2. The same evaluation is also computed for each 
feature selection and ranking measures defined in this section. Later, the proposed fuzzy-based composite measure is applied for improving the capabilities of the proposed model.

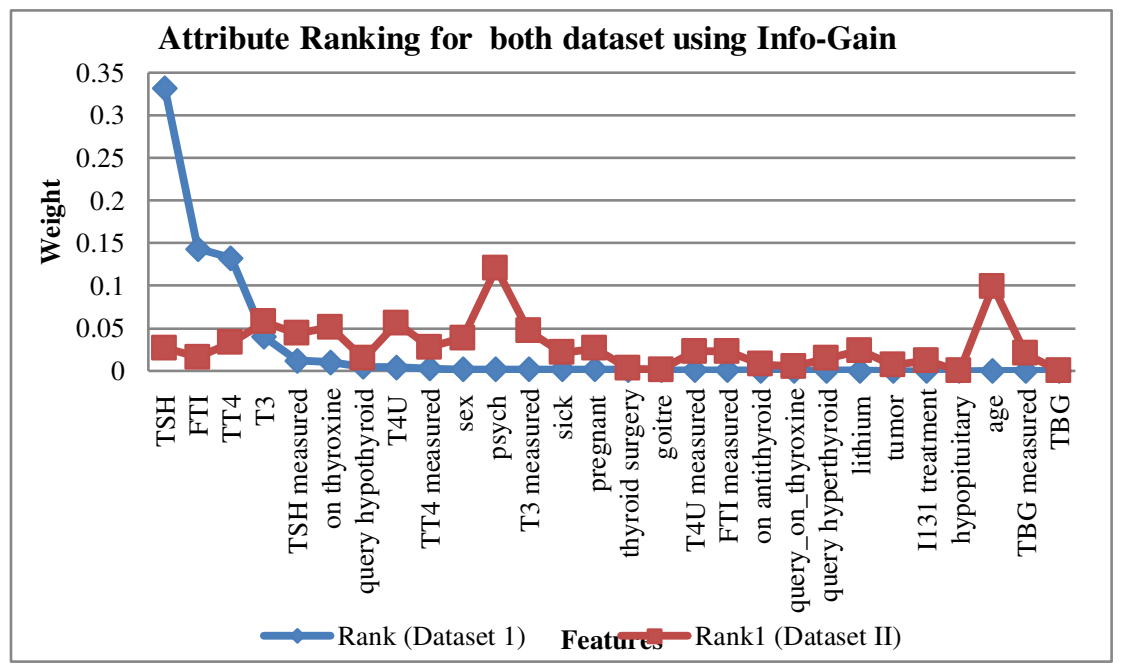

Figure 2: Attribute Ranking for both Datasets

Figure 2 is showing the attribute ranking evaluated for both datasets using the infogain ranking algorithm. The attributes are showing on X-axis, and the y-axis is showing the weights based on the info-gain measure. By observing weights for these two datasets, the most contributing and high ranked features are selected in this proposed model. For the rest of the feature rankers, the values are provided in Table 4.

\subsubsection{Gain-Ratio}

Gain ratio[12][34] is the improved form of information gain that uses the normalized information for feature evaluation. The gain ratio is capable of reducing the biasness against the large attributes. It uses a split information value based normalized analysis to process the features in partitions. The splition of the dataset (D) into $\mathrm{v}$ partitions is done, and relatively $\mathrm{v}$ values come in outcome for an attribute (Attr). The splitinfo evaluation is provided in Equation (5).

$$
\text { SplitInfo }_{A t t} \mathrm{r}(\mathrm{D})=-\sum_{i=1}^{v} \frac{\left|D_{i}\right|}{|D|} \times \log _{2}\left(\frac{\left|D_{i}\right|}{D}\right)
$$

The higher value of Splitinfo shows the uniformity obtained over the attributes, and lower splitinfo represents the existence of peaks with more number of tuples in some partitions. After obtaining the SplitInfo, the Gain-Ratio is computed using Equation (6). The attributes are selected with a higher ratio.

$$
\text { GainRatio(Attr) }=\frac{\operatorname{Gain}(\text { Attr })}{\text { SplitInfo(Attr })}
$$

Gain-Ratio values obtained for various features of the Thyroid and Hypothyroid 
dataset are provided in Table 4.

\subsubsection{ChiSquare}

Chi-Square[51][52] is the statistical test that measures the dependency of two variables. The coefficient of determination is applied by this measure to evaluate the similarities. The Chi-Square test is very effective in measuring the contribution of categorical or nominal data. This test identifies the mapping of the dependent variable respective to the class label. The ChiSquare score evaluation with the specification of $\mathrm{C}$ classes and $\mathrm{k}$ different values is provided through Equation (7). The datasets considered in this research are having a number of attributes with nominal values. The ChiSquare test has performed well to evaluate the weights for these categorical features.

$$
\chi^{2}=\sum_{i=1}^{k} \sum_{j=1}^{C} \frac{\left(n_{i j}-\mu_{i j}\right)^{2}}{\mu_{i j}}
$$

Where $\mathrm{C}$ is the number of class, $\mathrm{k}$ is the different values, $n_{i j}$ defines a number of samples with $\mathrm{i}^{\text {th }}$ value, and

$$
\mu_{i j}=\frac{\left(n_{* j} n_{i *}\right)}{n}
$$

Where $n_{i *}$ defines the number of samples that takes $\mathrm{i}^{\text {th }}$ value of a feature and $n_{* j}$ defines the number of samples that map to class $\mathrm{j}$. ChiSquare values obtained for various features of the Thyroid and Hypothyroid dataset are provided in Table 4.

\subsubsection{ReliefF}

ReliefF[12][50] is the feature selector which is used effectively for binary classification problem. The filter is sensitive to the interactions among the features. This filter evaluates the nearest neighbor difference for scoring the features. It generates the 'hit' and 'miss' based observations respective to class specification. The weighted score is evaluated by this filter. In this research, multi-class analysis is considered so that the filter has not provided satisfactory results.

\subsection{Proposed Fuzzy based Composite-Measure and Recommendation adaptive Feature Selection}

In this paper, composite-measure and recommendation based fuzzy rules are defined for identifying the most contributing features. For setting up the global adaption for thyroid disease, the measures are computed for Thyroid and Hypothyroid datasets for two and multi-class disease prediction. Table 4 shows the values obtained InfoGain, Gain Ratio, and ChiSquare measures for all features. The fuzzy rules based on Equation (9), (10), and (11) are applied for obtaining the significance of feature on a specific dataset. In Table 4, the significant feature values are highlighted in red color. The equations are applied separately on each dataset. Equation (9) shows the fuzzy transition of feature-values of InfoGain measure. The Equation shows the Significant and NonSignificant fuzzy values are obtained by incorporating the 
fuzzification on the InfoGain parameter. A large of values for a fuzzy parameter (a) between 0 (Min) and 0.181 (Max) are tested for evaluating the most gainful decision on Significant and NonSignificant Features. The analysis is done respectively to the removal of recommended features and accuracy achieved on the filtered dataset. After conducting deep experimentation, $\mathrm{a}=0.005$ confirmed the adaptation of generalized rule for all datasets. This generalized fuzzy value achieved significant results among all experimented values.

$$
\text { fInfoGain= } \left.\begin{array}{c}
\text { InfoGain } \geq a \\
\text { InfoGain }<a
\end{array}\right\} \begin{gathered}
\text { Significant } \\
\text { NonSignificant }
\end{gathered}
$$

Where $\mathrm{a}=.005$ is parameter value used for Optimized fuzzy decision

GainRatio is another measure included in the proposed fuzzy-based composite rule formation. Fuzzification is applied to the GainRatio parameter for transiting it to Significant and NonSignificant categories. The fuzzy rule is derived by incorporating a wide range of values between minimum and maximum values of the fuzzy parameter (a). After experimenting with the fuzzy rules on all datasets, the observations identified $\mathrm{a}=0.2$ achieved the most accurate and generalized results for all datasets. Equation (10) shows the fuzzification rule of GainRatio and obtained the fGainRatio as output.

$$
\text { fGainRatio } \left.=\begin{array}{l}
\text { GainRatio } \geq a \\
\text { GainRatio }<a
\end{array}\right\} \begin{gathered}
\text { Significant } \\
\text { NonSignificant }
\end{gathered}
$$

Where $\mathrm{a}=.02$ is parameter value used for Optimized fuzzy decision

The third measure used in this proposed composite-fuzzy rule-based Feature selection method is ChiSquare. Fuzzification is applied to computed ChiSq values for identifying the Significant and NonSignificant features. The analytical evaluation is applied to different values of a for observing the strength of filtered featureset. The experimentation is conducted a vast range of iterative values between min and max for genetic and parametric evaluation. The analytical results identified that $a=50$ identified a dominating featureset that can provide globally high accuracy. The fuzzy rule for evaluation of fChiSq values is shown in Equation (11).

$$
\left.\mathrm{fChiSq}=\begin{array}{l}
\text { ChiS } q \geq a \\
\text { ChiS } q<a
\end{array}\right\} \begin{gathered}
\text { Significant } \\
\text { NonSignificant }
\end{gathered}
$$

Where $\mathrm{a}=50$ is parameter value used for Optimized fuzzy decision

Each of the measures is fuzzified for all features of four datasets. The identified Significant values of each measure and feature are highlighted in pink boxes. Now, the Fuzzy AND and OR operations are applied to these Significant(SG) and NonSignificant(NSG) fuzzy values. This composite rule is applied to the fuzzy values of each dataset. This fuzzy rule computed each measure in terms of High, Medium, and Low contributing features. Equation (12) defines the fuzzy And-OR rule applied for obtaining the fuzzy values for these dataset-specific values. This rule is applied to fuzzy computed fInfoGai, fGainRatio, and fChiSq features respective to Significant and NonSignificant values. The generalized rule is defined by analyzing the Significant and NonSignificant values of each dataset and decided the degree of measure. This degree of measure is described in terms of Fuzzy High, Medium, and Low values. The transited generalized fuzzy values of InfoGain, Gain Ratio, and 
ChiSq measures are provided in Table 5. These generalized-fuzzy feature values are further combined with recommended values for selecting or rejecting the features.

$$
\text { GfMeasure } \left.=\begin{array}{c}
(S G \cap S G \cap S G \cap S G) \cup(N S G \cap S G \cap S G \cap S G) \\
(N S G \cap N S G \cap S G \cap S G) \\
\text { Otherwise }
\end{array}\right\}_{L}^{H}
$$

Here, GfMeasure is the generalized fuzzy measure applied on computed fuzzybased feature-measure values for each dataset, SG is the significant and NSG are the non-significant fuzzy-measure values for specific features and datasets.

Table 4: Evaluation of Compounding Fuzzy-Features of Proposed Model (4

\begin{tabular}{|c|c|c|c|c|c|c|c|c|c|c|c|c|}
\hline & $\begin{array}{l}\text { Info } \\
\text { Gain }\end{array}$ & $\begin{array}{l}\text { Info } \\
\text { Gain } \\
\end{array}$ & Info Gain & $\begin{array}{l}\text { Info } \\
\text { Gain } \\
\end{array}$ & Gain ratio & Gain ratio & Gain ratio & $\begin{array}{l}\text { Gain } \\
\text { ratio }\end{array}$ & ChiSq & ChiSq & ChiSq & ChiSq \\
\hline & $\begin{array}{l}\text { Thyroid } \\
\text { (2 Class) }\end{array}$ & $\begin{array}{l}\text { Thyroid } \\
\text { Class }\end{array}$ & $\begin{array}{l}\text { Hypo } \\
\text { throid } \\
\text { Class) }\end{array}$ & $\begin{array}{l}\text { Hypot } \\
\text { hyroid }\end{array}$ & $\begin{array}{l}\text { Thyroid } \\
\text { (2 Class) }\end{array}$ & $\begin{array}{l}\text { Thyroid } \\
\text { Class }\end{array}$ & $\begin{array}{l}\text { Hypo } \\
\text { thyroid } \\
\text { (2Class) }\end{array}$ & $\begin{array}{l}\text { Hypo } \\
\text { thyroid }\end{array}$ & $\begin{array}{l}\text { Thyroid } \\
\text { Class) }\end{array}$ & $\begin{array}{l}2 \text { Thyroid } \\
\text { Class }\end{array}$ & $\begin{array}{l}\text { Hypot } \\
\text { hyroid }(2 \\
\text { Class) }\end{array}$ & $\begin{array}{l}\text { Hypo } \\
\text { thyroid }\end{array}$ \\
\hline age & 0.0029 & 0.0354 & 0.0000 & 0.0000 & 0.0040 & 0.0211 & 0.0000 & 0.0000 & 37.7132 & 507.2721 & 0.000 & 0.0000 \\
\hline $\operatorname{sex}$ & 0.0057 & 0.0142 & 0.0019 & 0.0020 & 0.0063 & 0.0158 & 0.0021 & 0.0023 & 69.6357 & 164.3521 & 9.350 & 10.0891 \\
\hline $\begin{array}{ll}\text { on } & \text { thyr } \\
\text { oxine } & \\
\end{array}$ & 0.0061 & 0.1122 & 0.0064 & 0.0103 & 0.0107 & 0.1963 & 0.0119 & 0.0192 & 83.8215 & 2185.7691 & 24.79 & 30.1083 \\
\hline $\begin{array}{l}\text { query_- } \\
\text { on_th } \\
\text { yroxine }\end{array}$ & 0.0001 & 0.0012 & 0.0000 & 0.0005 & 0.0005 & 0.0101 & 0.0004 & 0.0050 & 0.8038 & 20.8661 & 0.210 & 1.3915 \\
\hline $\begin{array}{l}\text { on_ant } \\
\text { ithyroid_- } \\
\text { medication }\end{array}$ & 0.0001 & 0.0040 & 0.0005 & 0.0006 & 0.0014 & 0.0412 & 0.0051 & 0.0068 & 1.8812 & 225.8406 & 1.774 & 1.9268 \\
\hline sick & 0.0001 & 0.0046 & 0.0000 & 0.0017 & 0.0003 & 0.0199 & 0.0000 & 0.0074 & 0.7206 & 60.2428 & 0.012 & 5.5627 \\
\hline pregnant & 0.0081 & 0.0237 & 0.0016 & 0.0016 & 0.0888 & 0.2585 & 0.0154 & 0.0154 & 129.1594 & 885.8660 & 4.494 & 4.4938 \\
\hline $\begin{array}{l}\text { Thyroid } \\
\text { surgery }\end{array}$ & 0.0000 & 0.0080 & 0.0003 & 0.0011 & 0.0000 & 0.0724 & 0.0026 & 0.0106 & 0.0000 & 403.9375 & 1.173 & 3.2199 \\
\hline $\begin{array}{l}1131_{-} \\
\text {treatment }\end{array}$ & 0.0000 & 0.0029 & 0.0000 & 0.0000 & 0.0003 & 0.0221 & 0.0001 & 0.0004 & 0.5259 & 50.6536 & 0.049 & 0.2166 \\
\hline $\begin{array}{l}\text { query_hypo } \\
\text { thyroid }\end{array}$ & 0.0031 & 0.0055 & 0.0042 & 0.0046 & 0.0085 & 0.0152 & 0.0126 & 0.0136 & 42.2626 & 80.0069 & 28.081 & 33.3158 \\
\hline $\begin{array}{l}\text { query__ } \\
\text { hyper } \\
\text { thyroid }\end{array}$ & 0.0012 & 0.0053 & 0.0001 & 0.0004 & 0.0032 & 0.0144 & 0.0004 & 0.0013 & 16.0463 & 75.1535 & 0.6820 & 1.7656 \\
\hline Lithium & 0.0005 & 0.0011 & 0.0000 & 0.0002 & 0.0060 & 0.0131 & 0.0006 & 0.0041 & 5.5118 & 8.6092 & 0.1184 & 0.4802 \\
\hline Goiter & 0.0007 & 0.0015 & 0.0010 & 0.0010 & 0.0091 & 0.0202 & 0.0141 & 0.0141 & 7.3344 & 10.9123 & 2.8681 & 2.8681 \\
\hline Tumor & 0.0005 & 0.0024 & 0.0000 & 0.0001 & 0.0027 & 0.0139 & 0.0001 & 0.0005 & 6.3675 & 39.4790 & 0.0529 & 0.3650 \\
\hline $\begin{array}{l}\text { Hypopi } \\
\text { tuitary }\end{array}$ & 0.0005 & 0.0008 & 0.0000 & 0.0000 & 0.1554 & 0.2605 & 0.0087 & 0.0087 & 6.6543 & 21.4956 & 0.0836 & 0.0836 \\
\hline Psych & 0.0060 & 0.0074 & 0.0007 & 0.0019 & 0.0224 & 0.0277 & 0.0024 & 0.0069 & 60.7264 & 65.5323 & 3.0800 & 5.4499 \\
\hline $\begin{array}{l}\mathrm{TSH} \\
\text { measured }\end{array}$ & 0.0115 & 0.0134 & 0.0120 & 0.0120 & 0.0261 & 0.0302 & 0.0259 & 0.0259 & 119.8432 & 126.7671 & 34.192 & 34.1920 \\
\hline TSH & 0.1810 & 0.3130 & 0.2925 & 0.3318 & 0.1231 & 0.1799 & 0.5939 & 0.5245 & 2658.7448 & 5386.4985 & 2576.4 & 4242.6957 \\
\hline $\begin{array}{l}\mathrm{T} 3 \\
\text { measured }\end{array}$ & 0.0032 & 0.0232 & 0.0008 & 0.0019 & 0.0038 & 0.0269 & 0.0011 & 0.0026 & 39.8280 & 215.4355 & 4.0744 & 8.0043 \\
\hline
\end{tabular}
Datasets) 


\begin{tabular}{|c|c|c|c|c|c|c|c|c|c|c|c|c|}
\hline T3 & 0.1200 & 0.2358 & 0.0263 & 0.0398 & 0.0698 & 0.1332 & 0.0161 & 0.0308 & 1741.7800 & 4919.1134 & 191.34 & 463.7348 \\
\hline $\begin{array}{l}\mathrm{TT} 4 \_ \\
\text {measured }\end{array}$ & 0.0123 & 0.0129 & 0.0027 & 0.0031 & 0.0441 & 0.0464 & 0.0082 & 0.0092 & 108.7340 & 109.5107 & 10.647 & 11.1592 \\
\hline TT4 & 0.1293 & 0.2146 & 0.0836 & 0.1323 & 0.0633 & 0.0768 & 0.0525 & 0.0802 & 1891.5441 & 4201.5284 & 753.01 & 2226.7712 \\
\hline $\begin{array}{l}\mathrm{T} 4 \mathrm{U}_{-} \\
\text {measured }\end{array}$ & 0.0065 & 0.0081 & 0.0002 & 0.0007 & 0.0152 & 0.0187 & 0.0004 & 0.0014 & 71.7620 & 80.8035 & 0.954 & 4.8389 \\
\hline T4U & 0.0388 & 0.1428 & 0.0000 & 0.0041 & 0.0441 & 0.0660 & 0.0000 & 0.0049 & 587.2441 & 3618.0307 & 0.000 & 23.7767 \\
\hline $\begin{array}{l}\text { FTI_ } \\
\text { measured }\end{array}$ & 0.0065 & 0.0080 & 0.0002 & 0.0007 & 0.0152 & 0.0186 & 0.0004 & 0.0014 & 71.4091 & 80.0331 & 0.898 & 4.8012 \\
\hline FTI & 0.0993 & 0.1818 & 0.0864 & 0.1430 & 0.0742 & 0.0954 & 0.0416 & 0.0697 & 1485.5599 & 4174.4936 & 807.12 & 2434.9290 \\
\hline $\begin{array}{l}\text { TBG_- } \\
\text { measured }\end{array}$ & 0.0084 & 0.0086 & 0.0000 & 0.0000 & 0.0359 & 0.0370 & 0.0000 & 0.0000 & 76.7466 & 77.1923 & 0.0000 & 0.0000 \\
\hline TBG & 0.0000 & 0.0000 & 0.0000 & 0.0000 & 0.0000 & 0.0000 & 0.0000 & 0.0000 & 0.0000 & 0.0000 & 0.0000 & 0.0000 \\
\hline $\begin{array}{c}\text { Referral } \\
\text { source }\end{array}$ & 0.0203 & 0.1009 & 0.0040 & 0.0061 & 0.0136 & 0.0677 & 0.0026 & 0.0040 & 249.9336 & 1795.4592 & 17.27 & 24.8926 \\
\hline
\end{tabular}

The generalized fuzzy rules are incorporated on InfoGain, GainRatio, and ChiSQ features and obtained the GfInfoGain, GfGainRatio, and GfChiSq features. The fuzzy rules are implied to combine these features with Recommendation Weights. These fuzzy rules are shown in Table 5. Based on these rules, the features are most contributing features that are selected. In this proposed model, these selected features are further processed for building the machine learning model. These pruned and relevant features are further processed under the expansion stage described in section 3.2 .

Table 5: Selected-Features based on Recommendation and Fuzzy-Evaluation

\begin{tabular}{|c|c|c|c|c|c|}
\hline & $\begin{array}{c}\text { GfInfoGai } \\
n\end{array}$ & $\begin{array}{c}\text { GfGainRati } \\
\text { o }\end{array}$ & $\begin{array}{c}\text { GfChiS } \\
\text { q }\end{array}$ & $\begin{array}{l}\text { Recommendati } \\
\text { on }\end{array}$ & $\begin{array}{c}\text { Selecte } \\
\mathrm{d}\end{array}$ \\
\hline age & $\mathrm{L}$ & $\mathrm{L}$ & $\mathrm{L}$ & $\mathrm{L}$ & No \\
\hline sex & M & $\mathrm{L}$ & M & $\mathrm{L}$ & No \\
\hline on thyroxine & $\mathrm{H}$ & $\mathrm{L}$ & $\mathrm{M}$ & $\mathrm{L}$ & No \\
\hline $\begin{array}{l}\text { query_on } \\
\text { thyroxine }\end{array}$ & $\mathrm{L}$ & $\mathrm{L}$ & $\mathrm{L}$ & $\mathrm{L}$ & No \\
\hline $\begin{array}{l}\text { on_antithyroid } \\
\text { medication }\end{array}$ & $\mathrm{L}$ & $\mathrm{L}$ & $\mathrm{L}$ & $\mathrm{L}$ & No \\
\hline sick & $\mathrm{L}$ & $\mathrm{L}$ & $\mathrm{L}$ & $\mathrm{L}$ & No \\
\hline pregnant & $\mathrm{M}$ & $\mathrm{L}$ & M & $\mathrm{L}$ & No \\
\hline thyroid_surgery & $\mathrm{L}$ & $\mathrm{L}$ & $\mathrm{L}$ & $\mathrm{L}$ & No \\
\hline I131_treatment & $\mathrm{L}$ & $\mathrm{L}$ & $\mathrm{L}$ & $\mathrm{H}$ & Yes \\
\hline $\begin{array}{l}\text { query_hypothyroi } \\
\text { d }\end{array}$ & $\mathrm{L}$ & $\mathrm{L}$ & $\mathrm{L}$ & $\mathrm{L}$ & No \\
\hline $\begin{array}{l}\text { query_hyperthyro } \\
\text { id }\end{array}$ & $\mathrm{L}$ & $\mathrm{L}$ & $\mathrm{L}$ & $\mathrm{L}$ & No \\
\hline
\end{tabular}




\begin{tabular}{|c|c|c|c|c|c|} 
lithium & L & L & L & H & Yes \\
\hline Goiter & L & L & L & M & No \\
\hline Tumor & L & L & L & L & No \\
\hline hypopituitary & L & M & L & M & Yes \\
\hline psych & M & M & M & L & Yes \\
\hline TSH_measured & H & H & M & H & Yes \\
\hline TSH & H & H & H & H & Yes \\
\hline T3_measured & L & L & L & M & No \\
\hline T3 & H & H & H & M & Yes \\
\hline TT4_measured & M & M & M & H & Yes \\
\hline TT4 & H & H & H & H & Yes \\
\hline T4U_measured & M & L & M & M & Yes \\
\hline T4U & M & M & M & M & Yes \\
\hline FTI_measured & M & L & M & H & Yes \\
\hline FTI & H & H & H & H & Yes \\
\hline TBG_measured & M & M & M & L & Yes \\
\hline TBG & L & L & L & L & No \\
\hline referral_source & M & L & M & L & Yes \\
\hline
\end{tabular}

In this work, composite-fuzzy feature-measure is combined with recommended features for identifying the most significant features. These selected 15 features are listed in Table 5. These selected features include all the recommended features. This proposed feature selection measure is effectively better than other feature ranking methods. Top-ranked features obtained from some of the state-of-art feature selectors are listed in Table 6 . The observations show that these ranking methods not including all the recommended features. It shows that the earlier methods are not significant respective to expert concern. The analytical validation of the proposed feature ranking and filtration method against existing methods is provided in section IV. Moreover, these existing filters are dataset-specific. But the proposed composite and fuzzy measure based filter are generated over four datasets. This generic and global dataset can be applied to any other thyroid disease datasets.

Table 6: Analytical Evaluation of Proposed Filtration Measure (Top 15 Features/Thyroid Dataset)

\begin{tabular}{|c|c|c|}
\hline $\begin{array}{c}\text { Feature } \\
\text { Selection } \\
\text { Method }\end{array}$ & Selected Features & $\begin{array}{c}\text { Number of } \\
\text { Recommended } \\
\text { Features }\end{array}$ \\
\hline Info-Gain & psych, Age, T3, T4U, on thyroxine, T3 measured, & 2 \\
\hline
\end{tabular}




\begin{tabular}{|c|c|c|}
\hline & $\begin{array}{l}\text { TSH_measured, sex, TT4, TT4_measured, TSH, } \\
\text { pregnant, lithium, FTI_measured, T4U_measured }\end{array}$ & \\
\hline Gain-Ratio & $\begin{array}{l}\text { psych, lithium, pregnant, TT4_measured, } \\
\text { TSH_measured, I131_treatment, sick, } \\
\text { on_thyroxine, TBG_measured, } \\
\text { on_antithyroid_medication, T3_measured, }\end{array}$ & 3 \\
\hline Chi-Square & $\begin{array}{c}\text { psych, T4U,age, pregnant,T3,sick,lithium, } \\
\text { T3_measured, on_thyroxine,sex, TT4, } \\
\text { TSH_measured, TSH, TT4_measured, } \\
\text { FTI measured }\end{array}$ & 2 \\
\hline ReliefF & $\begin{array}{l}\text { T3_measured, on thyroxine, TSH_measured, } \\
\text { TT4_measured, TBG_measured, TBG, sex, } \\
\text { query_hyperthyroid, query_hypothyroid, } \\
\text { I131_treatment, psych, sick, T4U_measured, } \\
\text { FTI_measured, on_antithyroid_medication }\end{array}$ & 4 \\
\hline $\begin{array}{l}\text { Proposed } \\
\text { Composite } \\
\text { Fuzzy } \\
\text { Rule-based } \\
\text { method }\end{array}$ & $\begin{array}{c}\text { I131_treatment, lithium, hypopituitary, psych, } \\
\text { TSH_measured, TSH, T3, TT4_measured, TT4, } \\
\text { T4U_measured, T4U, FTI_measured, FTI, } \\
\text { TBG_measured, referral_source }\end{array}$ & 0 \\
\hline
\end{tabular}

\subsection{Expanded Feature Set Generation}

After applying the proposed Composite-Fuzzy measure based filter, first-Level ranked, and contributing features are derived. In the second level, the featureset is expanded by applying the statistical distance measures on each feature. The aggregative statistical measures such as Mean, Standard Deviation (SD), and Standard Error (SE) are computed on ranked features. These aggregative measures are combined with each selected feature using the distance measure. The aggregative features are defined specifically to observe the collective behavior of normal or nodisease instances. The computation of Mean for any features corresponding to Nodisease values is shown through Equation (13).

$$
\operatorname{Mean}(\mathrm{f})=\frac{\sum_{i=1}^{N} f_{i}}{N}
$$

Where $\mathrm{N}$ is the number of Normal or No-disease instances, $\mathrm{f}$ is the selected feature

The mean feature gives an estimate of average feature value, and the differencevalue analysis sets a window for identifying the instances that belong to the same class. The instances of the normal class will give better window mapping, whereas the instances of disease instances will be far away to this window. As the features are very relevant to the respective class, the mean-difference analysis provided significant and accurate results. Another aggregative measure taken in this work is Standard deviation (SD), and its estimation is shown through Equation (14). SD identifies the mean specific distribution of values over the features for Normal and disease instances over the training set. The deviation is analyzed and recorded for each 
instance. The deviation will map later on the testing set for obtaining the maximum mapping to the relative class.

$$
\mathrm{SD}(\mathrm{f})=\sqrt{\frac{\sum_{i=1}^{N}\left(f_{i}-\text { Mean }\right)^{2}}{N}}
$$

Where $\mathrm{N}$ is the number of Normal or Disease Instances

Standard Error (SE) is another measure that estimates the deviation over the sample and presents the difference as an error. Equation (15) represents the formula of SE computation.

$$
\operatorname{SE}(\mathrm{f})=\frac{S D}{\sqrt{N}}
$$

Where $\mathrm{N}$ is the number of Normal or Disease Instances

The distances features are computed on these aggregative features for expanding the featureset. The expansion is applied by considering the aggregative operation on label specific instances. On the testing set, the collective aggregate operation based distance measure is applied. The distance measure based computation is provided through Equation (16). When all the features are computed through this aggregative distance measure, a wider featureset is obtained. This dataset is further passed through the filtration stage for identifying the most contributing and relevant distance features. The second level filtration process and results are provided in the next subsection.

$$
\text { Exp_F = AggreF - fValue }
$$

Where, AggreF is the specific aggregative feature such as Mean, SD, and SE,

fValue is the actual feature value, Exp_F is the expanded new feature.

Fuzzy rules are employed on this expanded featureset for handling the uncertainties. The membership function is applied To the feature values for categorizing the feature data. The membership grading is evaluated based on the Equation (17). The function categorized the feature values to $(\mathrm{VH}, \mathrm{H}, \mathrm{N}, \mathrm{L}$, and $\mathrm{VL}$ ). The values of fuzzy parameters are computed by observing the trainingset values. $b$ and $\mathrm{c}$ are the minimum and maximum values of a feature value for Normal or NoDisease class. The value of a and $d$ is analyzed by observing the frequency ratio of instances in the category and set a clear cutoff value dynamically. The dynamic computation is applied for this feature specific transition, and the process is applied to each feature separately. After applying equation (17) on each feature of the expanded dataset, the normalized dataset is obtained. This fuzzy-normalized dataset is further passed through the proposed composite-measures and fuzzy filter for identifying the most relevant and contributing features. The generation of this ranked and filtered expanded featureset formation is described in the next subsection.

$$
\left.\begin{array}{rl} 
& E x F \geq a \\
a & <E x F \geq b \\
\text { fExFeature }=b & <E x F \geq c \\
c & <E x F \geq d \\
d & <E x F
\end{array}\right\} \begin{gathered}
V H \\
H \\
L \\
V L
\end{gathered}
$$

Where, a, b,c, d, and e are the decision parameters, ExF is the expanded feature value 


\subsection{Ranked and Filtered Featureset Formation (Level II)}

The expanded wider dataset is analyzed using the proposed composite-measure based fuzzy filter for generating a relevant and compact featureset. The functional behavior, parameters, and associated constraints are already described in section 3.2. According to this proposed filter, the fuzzification is applied to InfoGain, GainRatio, and ChiSq measures for isolating the Significant (SG) and NonSignificant (NSG). Table 7 contains the values of each of these measures for all four datasets. The SG features are highlighted by red color boxes. Further, the And-OR based fuzzy rules are applied for combining these measures and select the most relevant features.

Table 7 : Evaluation of Features using three filters on Expanded Datasets (4

Datasets)

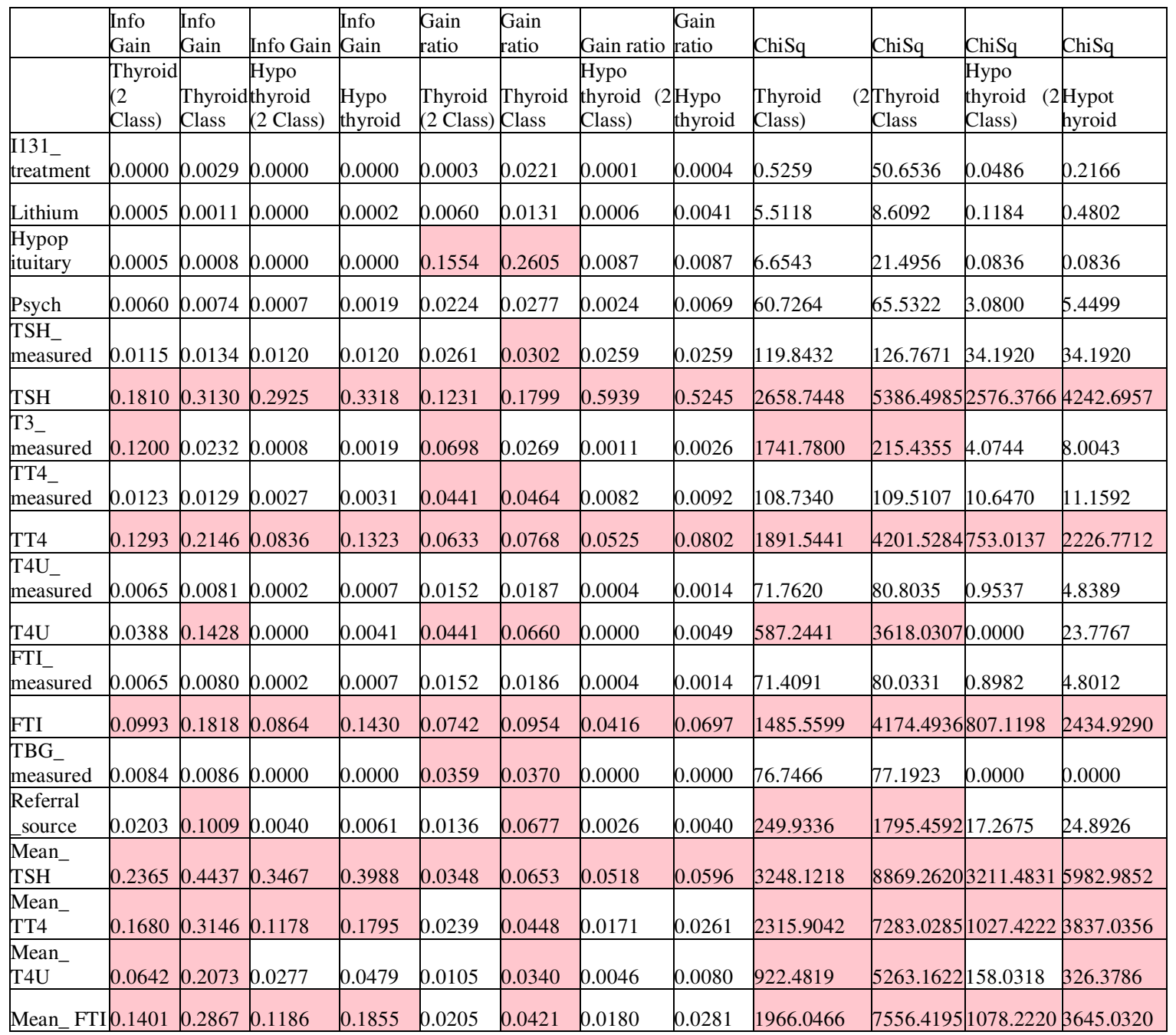




\begin{tabular}{|c|c|c|c|c|c|c|c|c|c|c|c|c|}
\hline STD_TSH & 365 & 0.4437 & 0.3467 & 3988 & 0.0348 & 0.0653 & 0.0518 & 0.0596 & 3248.1218 & 8869.2620 & 3211.4831 & 5982.9852 \\
\hline STD_TT4 & 0.1680 & 0.3146 & 0.1178 & 0.1795 & 0.0239 & 0.0448 & 0.0171 & 0.0261 & 231 & 7283.0285 & .4222 & 383 \\
\hline STD_T4U & 0. & 0.2073 & 0.0277 & 9 & 0.0105 & 0.0340 & 0.0046 & 0.0080 & 922 & 622 & & 326 \\
\hline STD_FTI & 0.1401 & 0.2867 & 0.1186 & 0.1855 & 0.0205 & 0.0421 & 0.0180 & 0.0281 & 1966 & 4195 & 220 & 320 \\
\hline SE_TSH & 0.2365 & 0.4437 & 0.3467 & 0.3988 & 0.03 & 0.0653 & 0.05 & 0.0596 & 324 & 620. & 831 & 852 \\
\hline SE_TT4 & 0.1680 & 0.3146 & 0.1178 & 0.1795 & 0.0239 & 0.0448 & 0.0171 & 0.0261 & 231 & 285 & 102 & 383 \\
\hline SE_T4U & 0.0642 & 0.2073 & 0.0277 & 0.0479 & 0.0105 & 0.0340 & 0.0046 & 0.0080 & 922. & 5263.1622 & & 326.3786 \\
\hline SE_FTI & 0.1401 & 0.2867 & 0.1186 & 0.1855 & 0.0205 & 0.0421 & 0.0180 & 0.0281 & 196 & 195 & 107 & 36 \\
\hline $\begin{array}{r}\text { TTest } \\
\text { TSH }\end{array}$ & 0.2365 & 0.4437 & 0.3467 & 0.3 & 0.0348 & 0.0653 & 0.0518 & 0.0596 & 324 & 886 & 321 & 598 \\
\hline $\begin{array}{r}\text { TTest } \\
\text { TT4 }\end{array}$ & 0.1680 & 0.3146 & 0.1178 & 0.1 & 0.0239 & 0.0 & 0.0 & 0.0261 & 231 & 728 & 102 & 38 \\
\hline $\begin{array}{r}\text { TTest } \\
\text { T4U }\end{array}$ & 0.0642 & 0.2073 & 0.0277 & 0.0479 & 0.0105 & 0.0340 & 0.0046 & 0.0080 & 922.4819 & 5263.1622 & 158.0318 & 326.3786 \\
\hline TTest & h 1401 & 0.2867 & 0.1186 & .1855 & .0205 & .0421 & 0.0180 & 0.0281 & 1966.0466 & 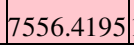 & 1078.2220 & 3645.0320 \\
\hline
\end{tabular}

Table 8 contains the fuzzy rules for selecting the most relevant, effective, and gainful features. Table contains the fuzzy values of InfoGain, GainRatio, and ChiSq measures. These fuzzy values are obtained by analyzing the And-OR operations applied to the SG/NSG values of each dataset. Based on the SG features and corresponding AND-OR relation among all datasets, the attribute-measure contribution is identified in terms of High $(\mathrm{H})$, Medium(M) and Low (L). Further, the And-OR based fuzzy rules are applied for combining these measures and select the most relevant features. Table 8 states the features identified after applying the Level-2 features. Both the training and testing datasets are expressed as these features, and the classifier is applied to this ranked and filtered dataset.

Table 8: Fuzzy-Evaluated Feature Selection on Expanded Feature Set

\begin{tabular}{|l|l|c|c|c|}
\hline & Info Gain & $\begin{array}{c}\text { Gain } \\
\text { Ratio }\end{array}$ & $\begin{array}{c}\text { ChiS } \\
\text { Q }\end{array}$ & $\begin{array}{c}\text { Select } \\
\text { ed }\end{array}$ \\
\hline I131_treatment & L & L & L & No \\
\hline lithium & L & L & L & No \\
\hline hypopituitary & L & M & L & No \\
\hline psych & L & L & L & No \\
\hline TSH_measured & L & L & L & No \\
\hline TSH & H & H & H & Yes \\
\hline T3_measured & L & L & M & No \\
\hline TT4_measured & L & M & L & No \\
\hline TT4 & H & H & H & Yes \\
\hline T4U_measured & L & L & L & No \\
\hline T4U & L & M & M & Yes \\
\hline
\end{tabular}




\begin{tabular}{|c|c|c|c|c|}
\hline FTI_measured & $\mathrm{L}$ & $\mathrm{L}$ & $\mathrm{L}$ & No \\
\hline FTI & $\mathrm{H}$ & $\mathrm{H}$ & $\mathrm{H}$ & Yes \\
\hline TBG_measured & $\mathrm{L}$ & $\mathrm{M}$ & $\mathrm{L}$ & No \\
\hline referral_source & $\mathrm{L}$ & $\mathrm{L}$ & $\mathrm{M}$ & No \\
\hline Mean_TSH & $\mathrm{H}$ & $\mathrm{H}$ & $\mathrm{H}$ & Yes \\
\hline Mean_TT4 & $\mathrm{H}$ & $\mathrm{L}$ & $\mathrm{H}$ & Yes \\
\hline Mean_T4U & $\mathrm{M}$ & $\mathrm{L}$ & $\mathrm{H}$ & Yes \\
\hline Mean_FTI & $\mathrm{H}$ & $\mathrm{L}$ & $\mathrm{H}$ & Yes \\
\hline STD_TSH & $\mathrm{H}$ & $\mathrm{H}$ & $\mathrm{H}$ & Yes \\
\hline STD_TT4 & $\mathrm{H}$ & $\mathrm{L}$ & $\mathrm{H}$ & Yes \\
\hline STD_T4U & $\mathrm{M}$ & $\mathrm{L}$ & $\mathrm{H}$ & Yes \\
\hline STD_FTI & $\mathrm{H}$ & $\mathrm{L}$ & $\mathrm{H}$ & Yes \\
\hline SE_TSH & $\mathrm{H}$ & $\mathrm{H}$ & $\mathrm{H}$ & Yes \\
\hline SE_TT4 & $\mathrm{H}$ & $\mathrm{L}$ & $\mathrm{H}$ & Yes \\
\hline SE_T4U & $\mathrm{M}$ & $\mathrm{L}$ & $\mathrm{H}$ & Yes \\
\hline SE_FTI & $\mathrm{H}$ & $\mathrm{L}$ & $\mathrm{H}$ & Yes \\
\hline TTest_TSH & $\mathrm{H}$ & $\mathrm{H}$ & $\mathrm{H}$ & Yes \\
\hline Ttest_TT4 & $\mathrm{H}$ & $\mathrm{L}$ & $\mathrm{H}$ & Yes \\
\hline Ttest_T4U & $\mathrm{M}$ & $\mathrm{L}$ & $\mathrm{H}$ & Yes \\
\hline Ttest_FTI & $\mathrm{H}$ & $\mathrm{L}$ & $\mathrm{H}$ & Yes \\
\hline
\end{tabular}

The proposed fuzzy filtered and expanded measure is applied for generating the dataset with the most relevant and contributing features. The functional process of expanded feature generation is provided in Algorithm I. The algorithm accepts the Labeled trainingset as input and returns the MLModel as output. The process of fuzzy-based feature filtration and expansion are described in the algorithm. The machine learning model is incorporated with a Dynamic weight adjustment based ELM classifier. The constraints and functional behavior of the classifier are described in the next subsection.

\section{Algorithm I: ExpendedFeaturedbasedMLModel}

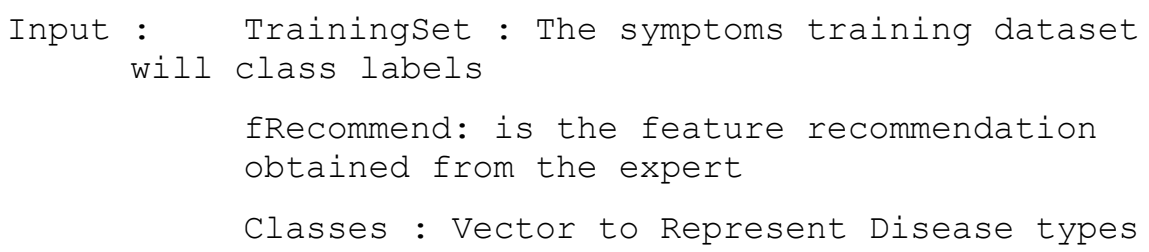


1. [InfoGain, GainRatio, ChiSq]=Ranking (TrainingSet, Classes) /*Apply Individual Ranking*/

2. [fInfoGain, fGainRatio, fChiSq]=Fuzzify(InfoGain, GainRatio, ChiSq) /*Generate the fuzzy measures on feature rankers*/

3. Ranking=CompositeMeasureFuzzyFilter (fInfoGain, fGainRatio, fChiSq, fRecommend) /*Identify the attribute ranking by mapping the attributes of training instances to different classes*/

4. FTrainingSet=ExtractedRankedDataset (Trainingset, Ranking) /*Extract the ranked features to form processing trainingset*/

5. $[$ Mean, SD, SE] = GenerateStats (FTrainingSet) $/{ }^{*}$ Collect the statistical measures for the filtered training set*/

6. DFTrainingset $=$ GenerateDistFeatures (FTrainingSet, [Mean, SD, SE]) /*Generate the distancestatistical features*/

7. ExTrainingset $=[$ FTrainingset DFTrainingset] /*Generate the expanded wider feature set* /

8. FWeightedSet=ApplyFuzzyRules (ExTrainingSet) /*Generate Fuzzy Rule processed weighted dataset*/

9. [InfoGain, GainRatio, ChiSq]=Ranking (FWeightedSet, Classes) /*Apply Individual Ranking*/

10. [fInfoGain, fGainRatio, fChiSq]=Fuzzify(InfoGain, GainRatio, ChiSq) /*Generate the fuzzy measures on feature rankers*/

11. Ranking=CompositeMeasureFuzzyFilter (fInfoGain, fGainRatio,fChiSq) /*Identify the attribute ranking by mapping the attributes of training instances to different classes*/

12. FTrainingSet=ExtractedRankedDataset (FWeightedSet, Ranking) / Apply composite fuzzy ranking*/

13. MLModel =ELM(FTrainingSet, Classes) /*Generate the ELM Model relative to Disease Classes*/

14 Return MLModel 


\subsection{Thyroid Classification}

Extreme Learning Machine (ELM) combines the features and capabilities of the neural network and support vector machine (SVM). The behavior of ELM adapts the functionality of feed-forward neural networks along with computational intelligence. It is an intelligent method that acquires the parameters analytically instead of manually tuned. The structure of ELM is composed of a single hidden layer on which the weights are transferred using Feed-Forward architecture. Initially, the weights are randomly generated, which are further updated based on the analysis performed on each interaction. In this work, the $\mathrm{N}$ samples of trainingset are processed with $\left(\mathrm{EFx}_{\mathrm{i}}\right.$, $\left.t_{i}\right) \in R^{n} \times R^{m}(i=1,2, \ldots N)$ expanded features and with class label specification. The additive $\mathrm{N}$ hidden nodes are the output vector. The activation functions us expressed through Equation (18).

$$
\sum_{i=1}^{N} \beta_{i} g\left(w_{i} E F x_{j}+b_{i}\right)=O_{j} \text { Where } \mathrm{j}=1,2,3, \ldots . \mathrm{N}
$$

Where, $\mathrm{w}_{\mathrm{i}}=\left[w_{i 1}, w_{i 2} \ldots . w_{i n}\right]^{T}$ defines the weights on input neurons and connect with hidden layer, $\beta_{i}=\left[\beta_{i 1}, \beta_{i 2} \ldots . \beta_{i m}\right]^{T}$ are the weights that connect the hidden and output layer, $b_{i}$ is the threshold limit

In ELM, the single layer of feed-forward network tries to reduce the difference between $O_{j}$ and $t_{j}$. and it can be represented as Equation (19).

$$
\sum_{i=1}^{N} \beta_{i} g\left(w_{i} E F x_{j}+b_{i}\right)=t_{j} \text { Where } \mathrm{j}=1,2,3, \ldots . \mathrm{N}
$$

With each successive iteration, the output is analyzed for identifying the weight updation. The objective of the functional process of ELM is to minimize the cost provided in Equation (20).

$$
\operatorname{Min}|\beta \mathrm{g}-\mathrm{T}|
$$

The proposed expanded, filtered, and fuzzy feature-based ELM model is applied on two and multi-class datasets. The analysis and comparative results are provided in the next section for validating the performance of the proposed model.

\section{Experimental Results}

In this paper, a Fuzzy-filtered and expanded feature processed ELM classifier is applied for thyroid disease classification. The composite-measure based fuzzy filter is combined with the expert recommendations to identify the contributing features. The statistical-distance measure is applied to continuous features to expand the featureset. The transformation of continuous features to nominal form is done using fuzzy rules. At the second level, the composite-measure based fuzzy filter is applied on expanded featureset to identify the processing features. In the final stage, the ELM classifier is applied to identify the class of thyroid disease. In this section, the experimentation and analysis results are derived to verify the significance of the proposed model. The section contains the dataset description, parameter exploration, and comparative results. 


\section{Datasets}

The proposed model is applied to hypothyroid[39] and thyroid-L7[42] datasets. The hypothyroid[39] dataset is described by 30 attributes, including the symptoms, history, and hormones based measures. The dataset is having 3772 instances described by 23 discrete and seven continuous attributes. The instances of the dataset are categorized by four classes, including no-thyroid and three disease forms. The thyroid-C7[42] is the other dataset with 30 disease descriptive features and six disease classes. The dataset has 9172 instances. In this section, the proposed filteredexpanded featureset is processed by the ELM classifier for generating the analytical results. The comparative observations are taken against Naïve Bays, RBF Network, Decision Tree, Decision Table, Random Forest, and Multilayer Perceptron methods. Each classification method is validated using the 10 -fold method. The results of existing methods are taken by processing the original dataset. The comparative analysis is obtained in terms of classification accuracy, precision rate, recall rate, MAE, and RMSE parameters.

\subsection{Analysis Parameters}

\subsubsection{Accuracy:}

Accuracy is defined as the ratio between the correctly classified instances and the total number of instances in the testingset.

$$
\mathrm{Acc}=\frac{T P+T N}{T P+T N+F P+F N}
$$

Where, $\mathrm{TP}=$ True Positive, $\mathrm{TN}=$ True Negative, $\mathrm{FP}=$ False Positive, $\mathrm{FN}=$ False Negative

\subsubsection{MAE:}

MAE is the average magnitude of the errors in a set of predictions. Equation (22) represents the computation of MAE.

$$
\mathrm{MAE}=\frac{\sum_{i=1}^{N}\left|A_{c}-P_{c}\right|}{N}
$$

Where Ac is the actual class, $\mathrm{Pc}$ is the predicted class, $\mathrm{N}$ is the number of instances in the testingset

\subsubsection{RMSE:}

Root Mean Square Error (RMSE) is the standard deviation based prediction error. The evaluation of RMSE is shown in Equation (23)

$$
\mathrm{RMSE}=\sqrt{\frac{\sum_{i=1}^{N}\left(A_{c}-P_{c}\right)^{2}}{N}}
$$


Where $\mathrm{Ac}$ is the actual class, $\mathrm{Pc}$ is the predicted class, $\mathrm{N}$ is the number of instances in the testingset

\subsubsection{Precision:}

Precision is the extension of accuracy feature that defines the ratio of true positives and total positives. Total positive includes True and false positives. False Positives are the instances that are wrongly labeled as Positive. The formula of precision is shown in Equation (24).

$$
\text { Precision }=\frac{T P}{T P+F P}
$$

Where, $\mathrm{TP}=$ True Positive, FP=False Positives

\subsubsection{Recall:}

Recall defines the ratio of correctly recognized instances and total instances in the actual class. The total instances in actual class are defined by True positive and False negatives. Equation (25) shows the Equation of Recall.

$$
\text { Recall }=\frac{T P}{T P+F N}
$$

Where TP=True Positives, FN=False Negative

\subsection{Thyroid L-7 Dataset: Disease Existence Analysis}

The first experimentation of the proposed filtered-Expanded featureset based ELM model is conducted on the Thyroid L-7 dataset to identify the positive and negative existence of the disease. The dataset contains 9172 instances, including 7052 Negative and 2120 Positive instances. Dataset has 29 features. After performing twolevel filtration and expansion of the proposed model, the dataset contains 21 features. The ELM classifier is applied to this fuzzy transited dataset for recognizing the appropriate class. The experimentation is conducted using the 10 -fold method by defining the $10 \%$ testing set. The confusion matrix in Table 9 shows the prediction results for each class. The observations show that the 6960 negative instances are predicted correctly out of 7052 instances, and 2109 positive instances are predicted correctly. The proposed model achieved $98.88 \%$ accuracy. The comparative analysis against state-of-art classifiers is provided in Figures 3, 4, and 5.

Table 9: Confusion Matrix

\begin{tabular}{|c|c|c|c|}
\hline \multicolumn{2}{|c|}{$\mathrm{N}=9172$} & \multicolumn{2}{c|}{ Predicted } \\
\cline { 3 - 4 } \multicolumn{2}{|c|}{ Actual } & Negative & Positive \\
\cline { 2 - 4 } & Positive & 6960 & 92 \\
\hline
\end{tabular}

Figure 3 shows the performance analysis in terms of the accuracy parameter. The comparative analysis of the proposed method is provided against NaiveBayes, MLP, 
RBFNetwork, Decision Table, J48, and Random Forest classifiers. The bar graph shows that the proposed model outperformed all the classifiers with a maximum of $98.88 \%$ accuracy. RBF network is the least performer with $76.89 \%$ accuracy. J48 achieved a closer accuracy to the proposed model of $96.83 \%$ rate.

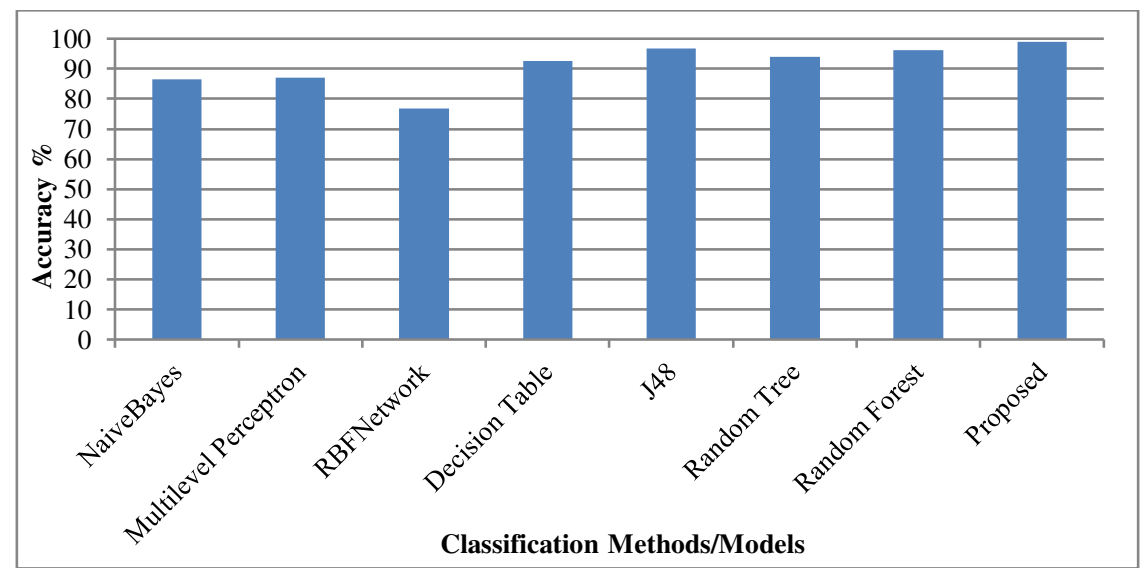

Figure 3: Accuracy analysis (2-Classes)

Another comparative evaluation to validate the proposed model is provided in terms of Error analysis. The classification error is computed in terms of MAE and RMSE parameters. The classification model with the least error rate will be considered more reliable. The bar graph in Figure 4 shows the MAE and RMSE values obtained for the proposed model are 0.0216 and 0.104 . Among the state-of-art classifiers, J48 achieved the least MAE and RMSE of 0.0448 and 0.1645 . The bar graph shows that the proposed model outperformed all the existing classifiers with a larger difference.

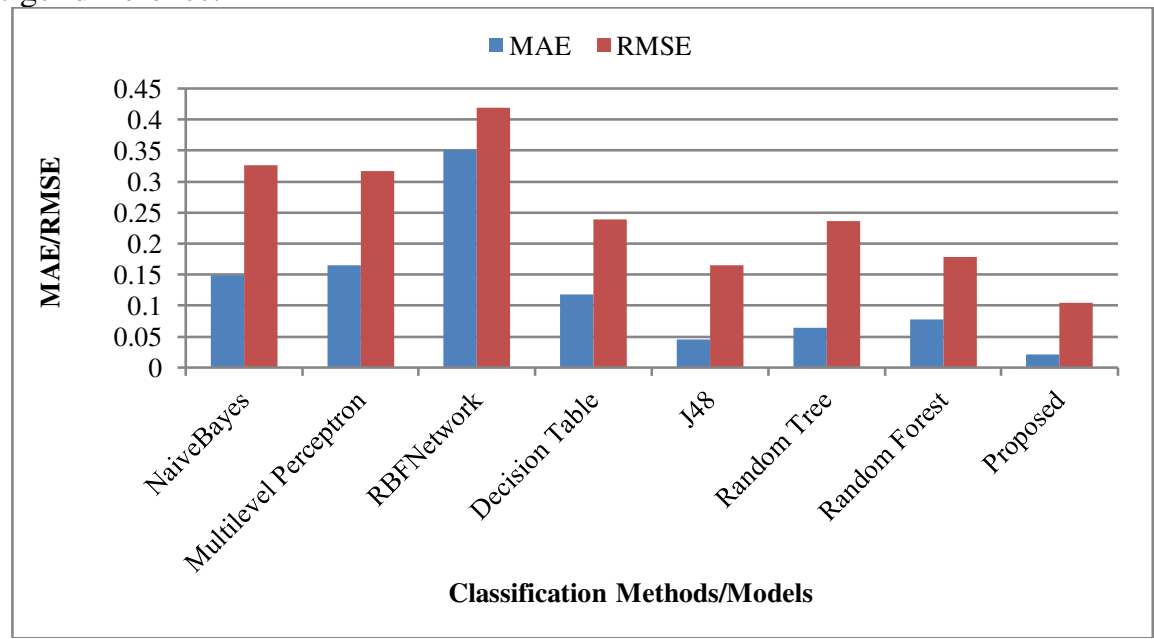

Figure 4: Error Analysis (2-Classes) 
The relevancy and accurate classification analysis are conducted in this work using Precision and Recall parameters. Figure 5 verifies the significance of the proposed model as the maximum precision and recall of 0.989. Among the state-of-art classifiers, RBFNetwork is the least performer with 0.591 and 0.769 rate of Precision and Recall. J48 achieved the 0.969 and 0.968 rates of Precision and Recall values, but still, it is quite lesser than the proposed model.

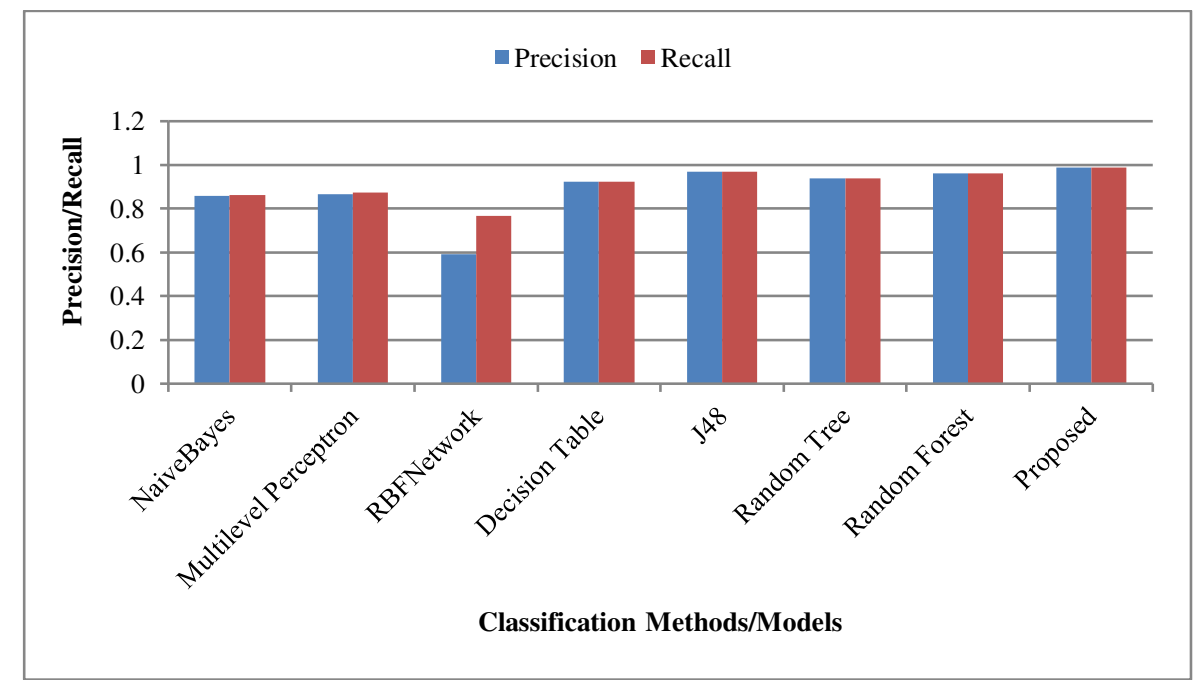

Figure 5: Precision/Recall Analysis (2-Classes)

The analytical and comparative observations obtained in this section verify that the proposed model is highly significant and reliable than other existing methods. The proposed model accurately identifies the existence of the disease in an individual.

\subsection{Thyroid L-7 Dataset: Disease Class Analysis (6 Classes)}

In experiment II, the analysis is conducted to recognize the disease class. The experimentation is conducted on the same Thyroid L-7 dataset that has 9172 instances and 29 features. In the proposed filtered-expanded form, the dataset has 21 features. The dataset is defined with Six classes. The dataset is having 7052 instances of health diseases. The dataset contains 2120 disease instances, including 883 of Hyperthyroid, 410 of Binding_protein, 346 of Replacement_Theory, 448 of General_Health, and 33 of antithyroid treatment. The proposed model is employed over this data for identifying the appropriate disease class. The confusion matrix obtained from the classification results is provided in Table 10. The results identified that most of the disease category and negative instances are identified accurately. The section also verified the performance against other state-of-art methods in terms of accuracy, error, and relevancy measures. 
Table 10: Confusion Matrix (6 Classes)

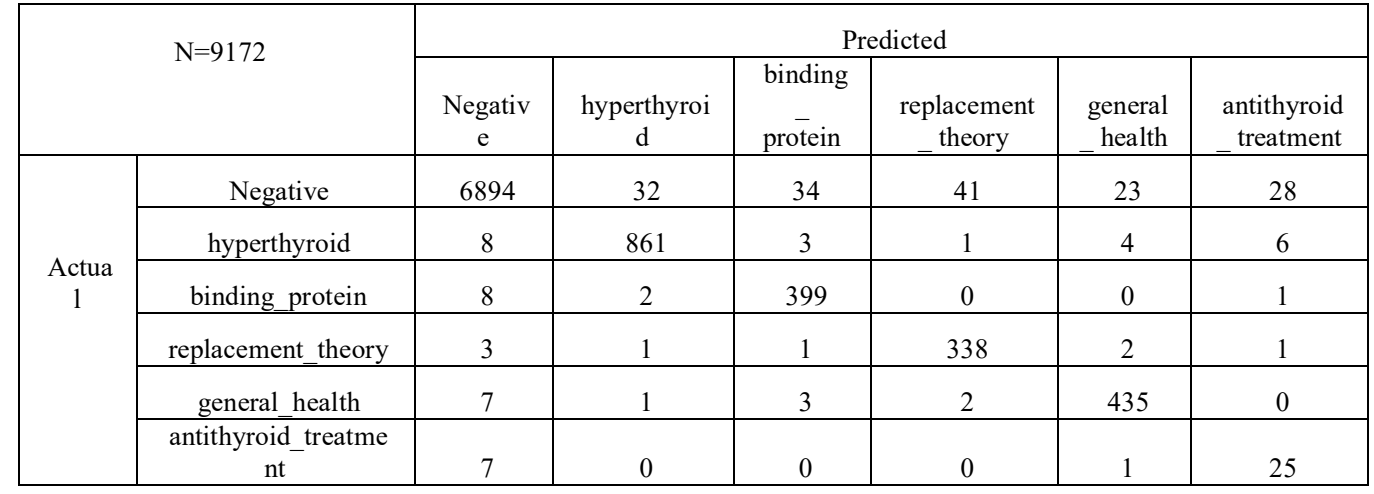

Figure 6 shows the performance verification of the proposed model against Naïve Bayes, MLP, RBFNetworks, Decision Table, Random Tree, Random Forest, and J48 classifiers. The bar graph identified that the proposed model claimed $97.60 \%$ accuracy, that is $1.5 \%$ higher than the best performer among existing classifiers.

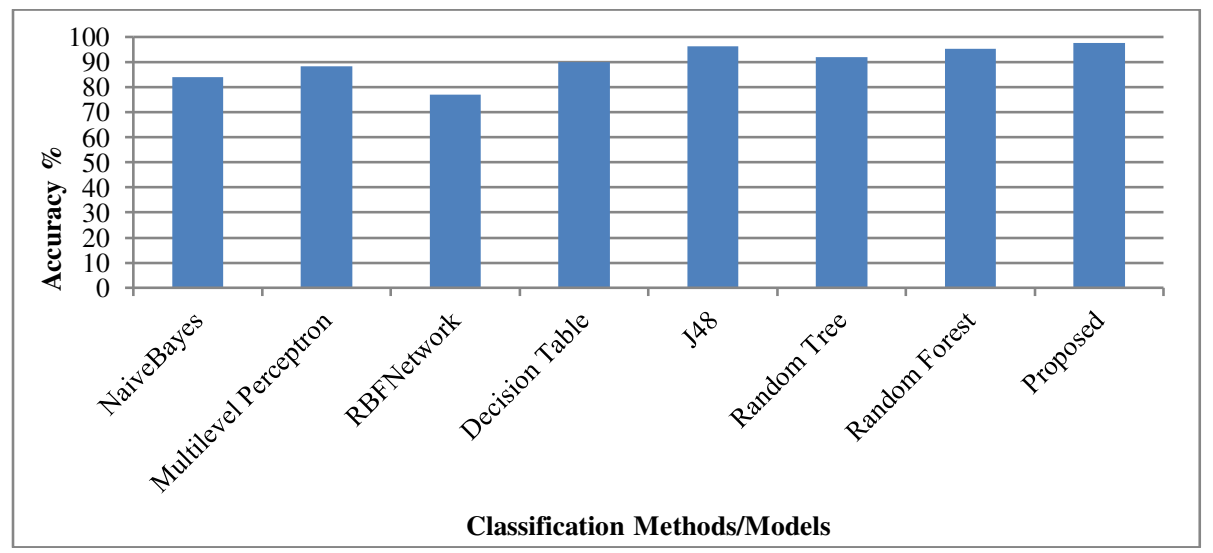

Figure 6: Accuracy analysis on Disease Class (6-Classes)

The error based comparative analysis of the proposed model is taken in terms of MAE and RMSE parameters. The lesser error rate identifies the reliable and effective classification of disease. Figure 7 shows the RMSE and MAE based comparative evaluation. The bar graphs that the proposed model claimed MAE $=0.0135$ and RMSE $=0.0823$. J48 achieved the effective MAE and RMSE of 0.0182 and 0.1056, which is even quite higher than the proposed model. It shows that the proposed model recorded a significantly lesser error rate than existing classifiers. 


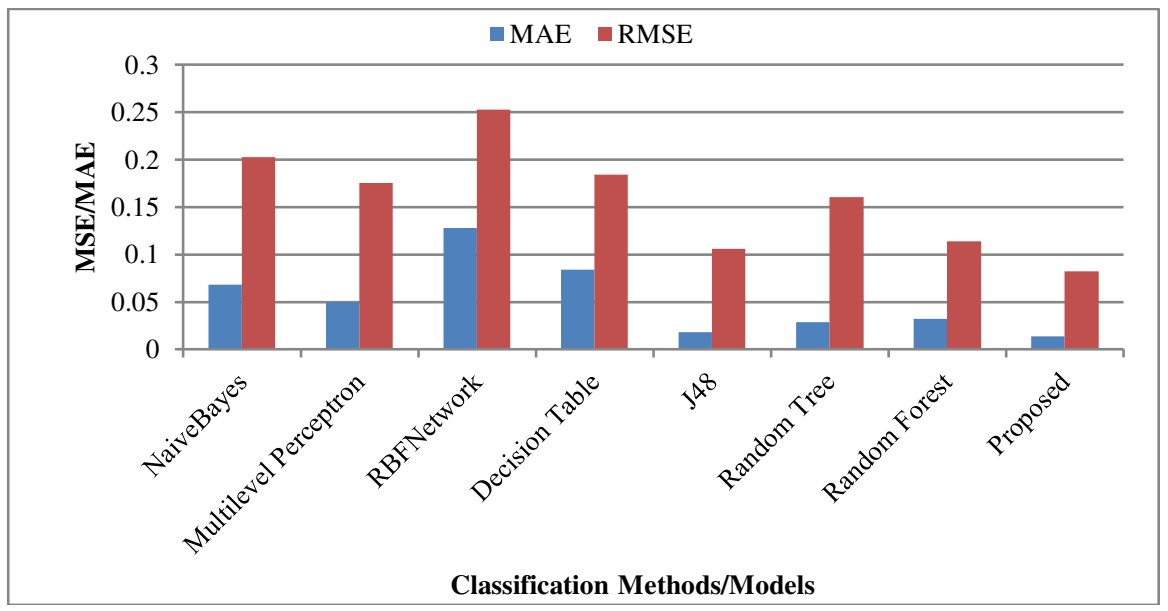

Figure 7: Error analysis on Disease Class (6-Classes)

The relevancy analysis of the proposed model is conducted based on Precision and Recall parameters. Figure 8 shows the comparative analysis against existing classifiers. The bar graphs show that the proposed model achieved the precision rate of 0.979 and recall rate of 0.976 . Among the existing methods, RBFNetwork is the least performer, and J48 is the best performer with 0.961 precision and recall rates. The results verified the higher performance of the proposed model against state-of-art methods.

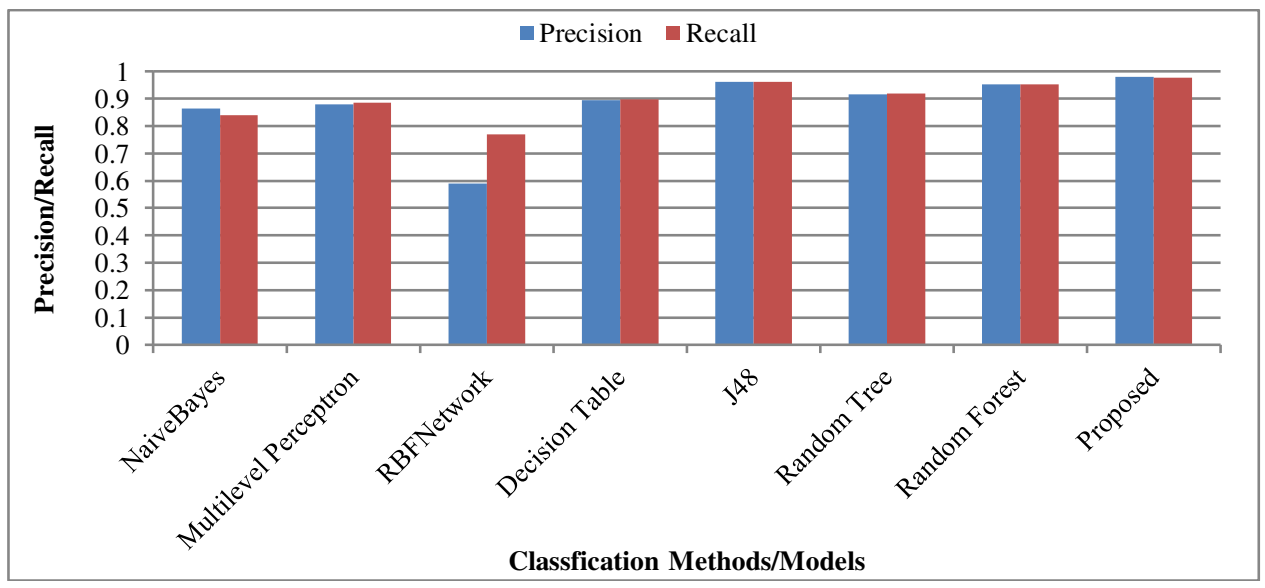

Figure 8: Precision and Recall Analysis (6-Classes)

The results provided in this section verify that the proposed model is effective for multi-class thyroid prediction. The proposed filtered-expanded and ELM classifierbased method ensured the reliable and effective results for multi-class prediction against existing classifiers. 


\subsection{Thyroid L-7 Dataset: Disease Subclass Analysis (18-Classes)}

Another Experiment is conducted on the Thyroid L-7 dataset for subclass disease classification. The dataset is having 9172 instances, 29 features, and 18 classes. The performance analysis against existing approaches is conducted using Accuracy, Error, and relevancy parameters. The accuracy-based analysis results are provided in Figure 9. The analysis results show the Decision Tree, Decision Table, Random Forest, and Random Tree methods achieved the significant accuracies of 95.9\%, 89.38\%, $95.15 \%$, and $91.46 \%$. The proposed expanded and filtered features based model achieved the most significant results, with $96.25 \%$ accuracy.

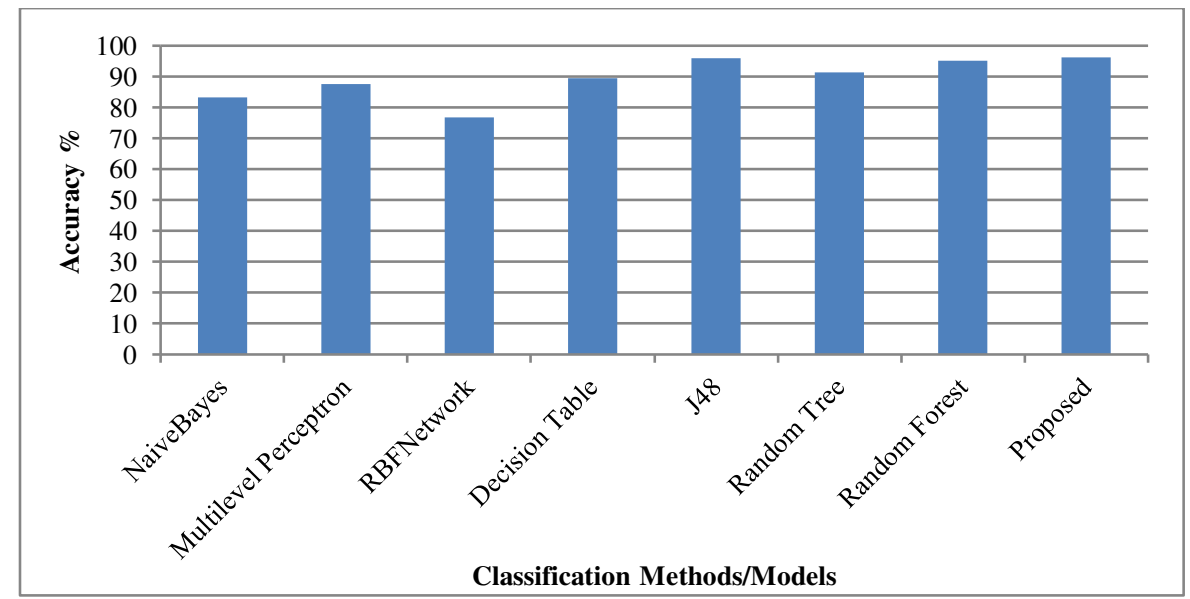

Figure 9: Accuracy Analysis (18 Classes)

The error based analysis of the proposed model is provided in Figure 10 using MAE and RMSE parameters. The figure shows that the proposed classification model achieved the least MAE and RMSE with .0067 and 0.0583 values. The error features of this model are quite lesser than the MAE and RMSE values of existing classifiers. Among existing methods, J48 achieved the most significant error features with 0.0063 MAE and 0.0622 RMSE. Even the MAE value of J48 is lesser than the proposed model, but it is not effective for RMSE and accuracy parameters. It signifies that the overall performance of the proposed model is better than all existing methods. 


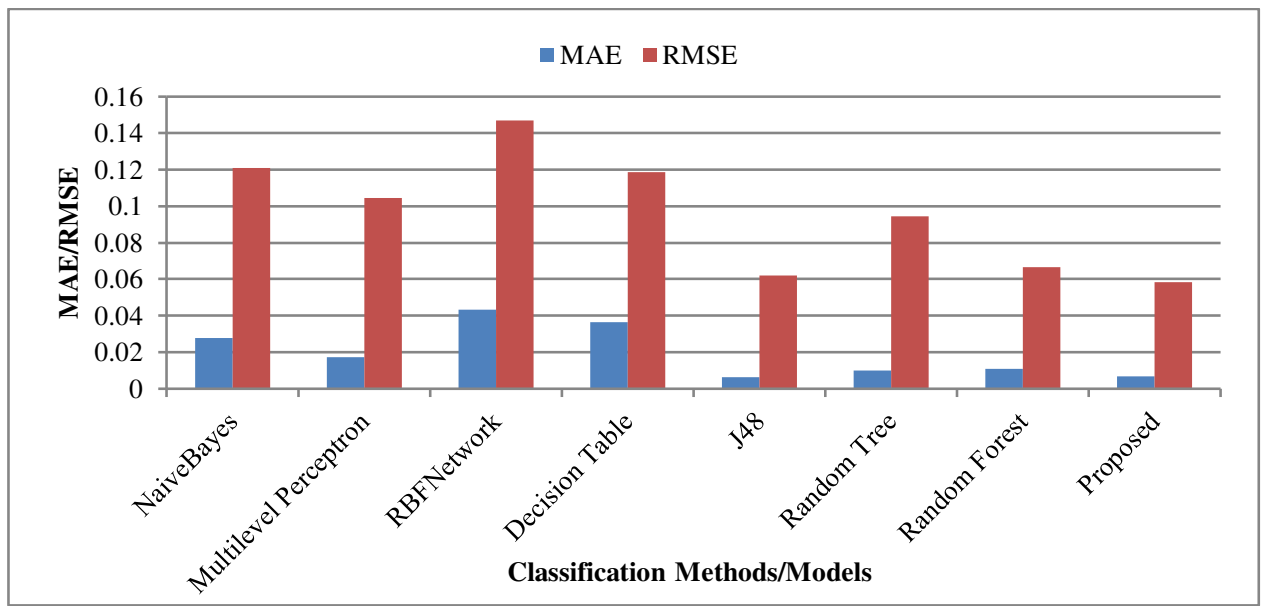

Figure 10: Error Analysis (18 Classes)

The relevancy based analysis for subclass prediction on thyroid dataset is provided in Figure 11. The figure shows that the proposed model gained the maximum relevancy with 0.961 precision rate and 0.962 recall rate. J48 and Random forest classifiers also achieved significant results with $0.958,0.949$ precision rate, and $0.959,0.951$ recall rates, respectively.

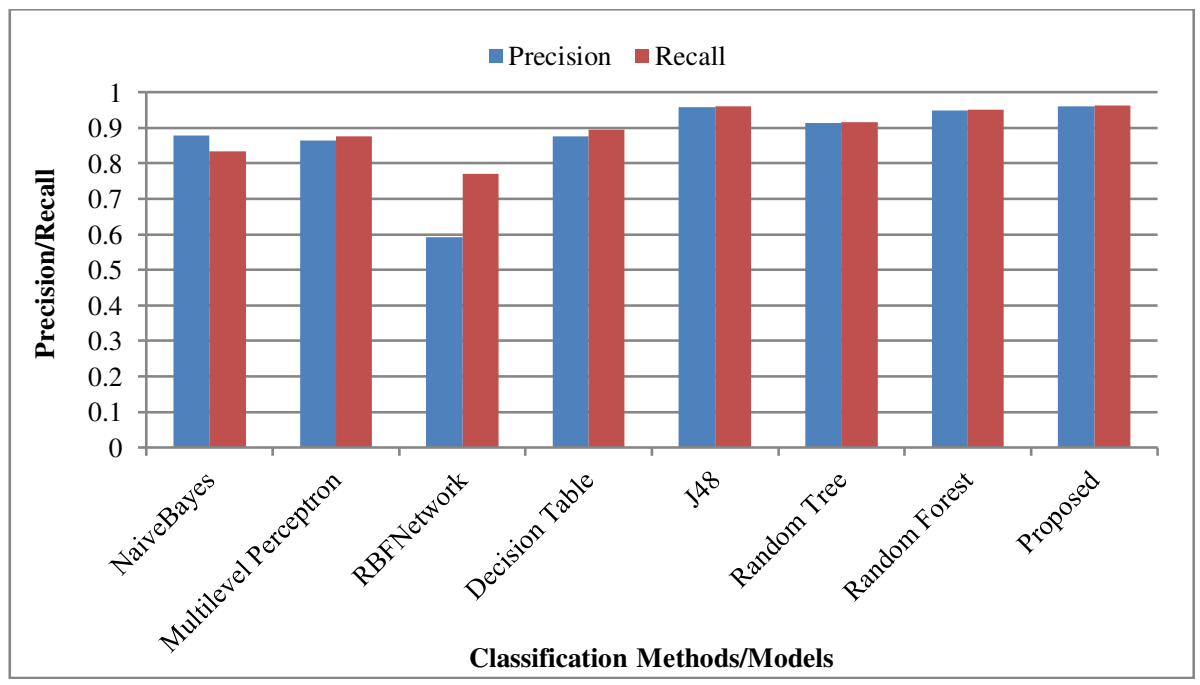

Figure 11: Precision/Recall Analysis (18 Classes)

The accuracy, relevancy, and error based evaluation results ensure the high performance and reliability achieved by this proposed model. The proposed model accurately predicts the disease, disease class, and subclass. For multi-class thyroid prediction, the proposed model achieved the most significant and reliable results. 


\subsection{Hyperthyroid Dataset: Disease Existence Analysis}

Another experiment in this research is conducted on the Hyperthyroid dataset. The dataset has 3772 instances and 29 features. In this section, the analysis results on disease existence are provided in comparison with existing methods. The dataset is having 3481 negative and 291 positive instances. The proposed filtered and expanded featureset based method is applied for identifying the disease existence over the dataset. The analytical evaluation of the proposed model is provided in terms of the Confusion matrix. Table 11 shows the confusion matrix results that show out of 3481 negative instances 3458, and out of 291 positive instances, 290 instances are predicted accurately. It shows that the proposed model achieved significant and reliable results. The comparative analysis against other state-of-art methods is provided in terms of accuracy, error, and relevancy parameters.

Table 11: Confusion Matrix (2 Classes)

\begin{tabular}{|c|c|c|c|}
\hline \multicolumn{2}{|c|}{} & \multicolumn{2}{c|}{ Predicted } \\
\cline { 3 - 4 } \multicolumn{2}{|c|}{$\mathrm{N}=3772$} & Negative & Positive \\
\hline \multirow{3}{*}{ Actual } & Negative & 3458 & 23 \\
\cline { 2 - 4 } & Positive & 1 & 290 \\
\hline
\end{tabular}

Figure 12 shows the comparative evaluation and validation of the proposed method against state-of-art classifiers analysis in terms of accuracy parameter. The results are provided against NaiveBayes, MLP, RBFNetwork, Decision Table, J48, and Random Forest classifiers. The bar graph shows that the proposed model outperformed all the existing classifiers and achieved $99.36 \%$ accuracy. Out of existing classifiers, the RBF network achieved the least accuracy of $95.0424 \%$. Decision table, Random forest, and J48 also achieved accuracy over 99\%, but still, the accuracy rate is lesser than the proposed model. It verifies the proposed model achieved a higher accuracy than existing classifiers.

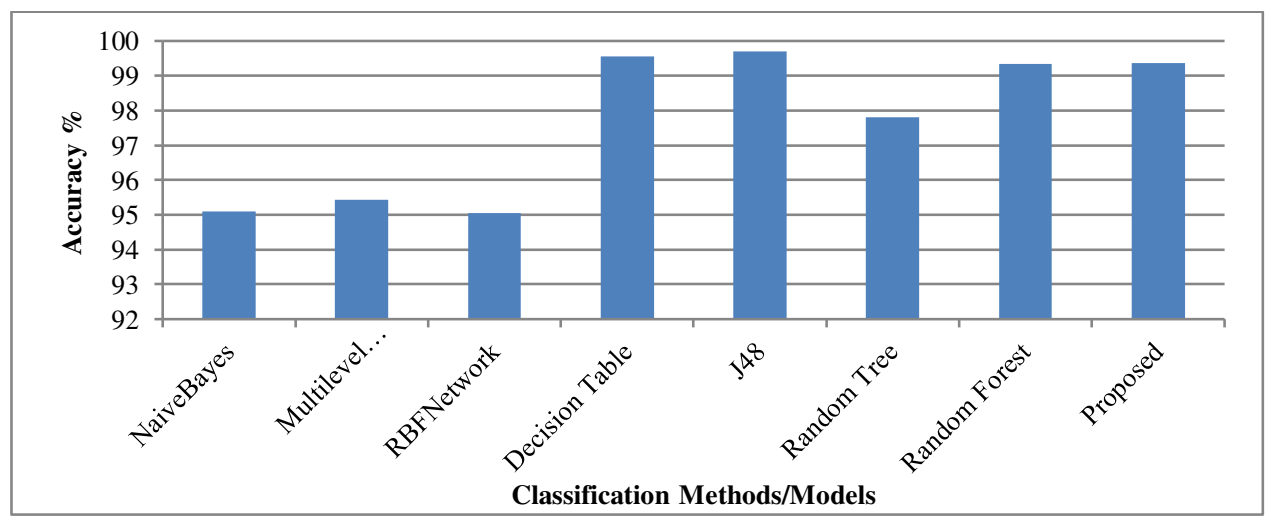

Figure 12: Accuracy Analysis (2 Classes) 
Error feature-based comparative analysis is provided in Figure 13 for validating the performance of the proposed model. The classification error is an analysis using MAE and RMSE parameters. The proposed classification model achieved the effective classification results with significant MAE and RMSE results with 0.0118 and 0.0771. J48 achieved the least classification error with 0.0051 MAE and 0.054 RMSE results. But overall results, including accuracy, relevancy, and error rate, the proposed model outperformed all the existing classifiers.

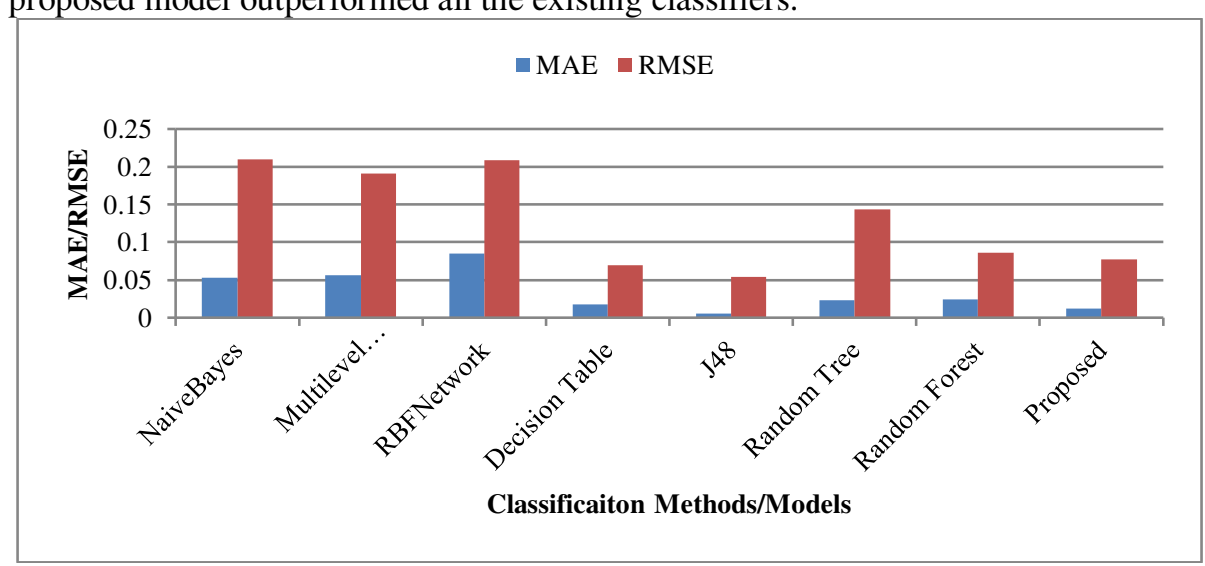

Figure 13: Error Analysis (2 Classes)

Precision and Recall parameters define the relevancy based analysis and the comparative results for these parameters are provided in Figure 14. The bar graph shows that the proposed model achieved significant precision and recall rate with values .994. Random Forest, Decision Tree, and Decision Tree achieved a better relevancy rate, but the accuracy of these methods is lesser than the proposed model. The combined results identified that the proposed model achieved more significant, relevant, and accurate results.

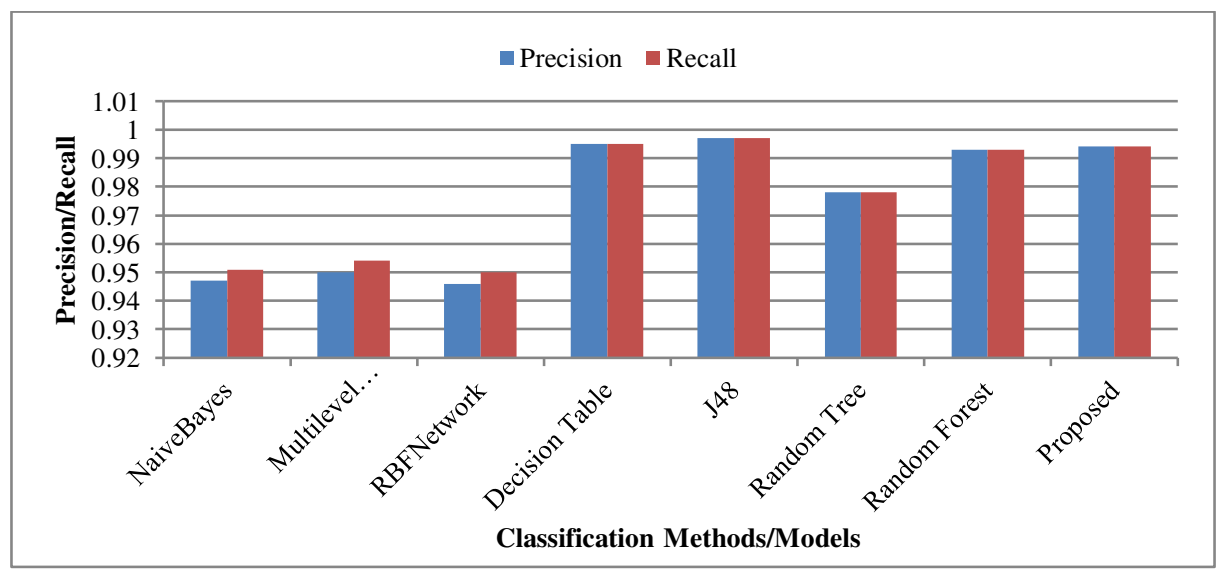

Figure 14: Precision/Recall Analysis 


\subsection{Hyperthyroid Dataset: Disease Class Analysis (4 Classes)}

Another disease class-based analysis is conducted on the Hyperthyroid dataset. In this multi-class prediction based experiment, the dataset is having four main classes called Negative, Compensated Hypothyroid, Primary Hypothyroid, and Secondary Hypothyroid. The proposed expanded and filtered featureset based ELM model is applied for effective, reliable, and accurate results. The class-specific prediction results are provided in Table 12 in the form of a confusion matrix. The confusion matrix shows that the proposed model recognized the health_patient instances 3473 out of 3481 records accuracy. In the same way, for Compensated hypothyroid, 193 out of 194 and for primary hypothyroid 94 out of 95 instances are recognized accurately. The confusion matrix shows that the proposed model achieved significant and effective results. Further, the comparative analysis against existing classifiers is provided against error, relevancy, and accuracy parameters.

Table 12: Confusion Matrix (4 Classes)

\begin{tabular}{|c|c|c|c|c|c|}
\hline \multirow{2}{*}{\multicolumn{2}{|c|}{ N=3772 }} & \multicolumn{4}{c|}{ Predicted } \\
\cline { 2 - 6 } & negative & $\begin{array}{c}\text { compensated } \\
\text { hypothyroid }\end{array}$ & $\begin{array}{c}\text { primary } \\
\text { hypothyroid }\end{array}$ & $\begin{array}{c}\text { secondary } \\
\text { hypothyroid }\end{array}$ \\
\hline \multirow{4}{*}{ Actual } & negative & 3473 & 4 & 4 & 0 \\
\cline { 2 - 6 } & $\begin{array}{c}\text { compensated } \\
\text { hypothyroid }\end{array}$ & 1 & 193 & 0 & 0 \\
\cline { 2 - 6 } & primary hypothyroid & 0 & 1 & 94 & 0 \\
\cline { 2 - 6 } & $\begin{array}{c}\text { secondary } \\
\text { hypothyroid }\end{array}$ & 2 & 0 & 0 & 0 \\
\hline
\end{tabular}

Accuracy based comparative analysis is provided in Figure 15 for analyzing the performance and reliability of the proposed model against existing classifiers. The graphs show that the proposed model achieved maximum accuracy with $99.68 \%$. Decision Table, J48, and Random forest also achieved the accuracy over 99\%, even though the proposed model achieved the maximum prediction rate.

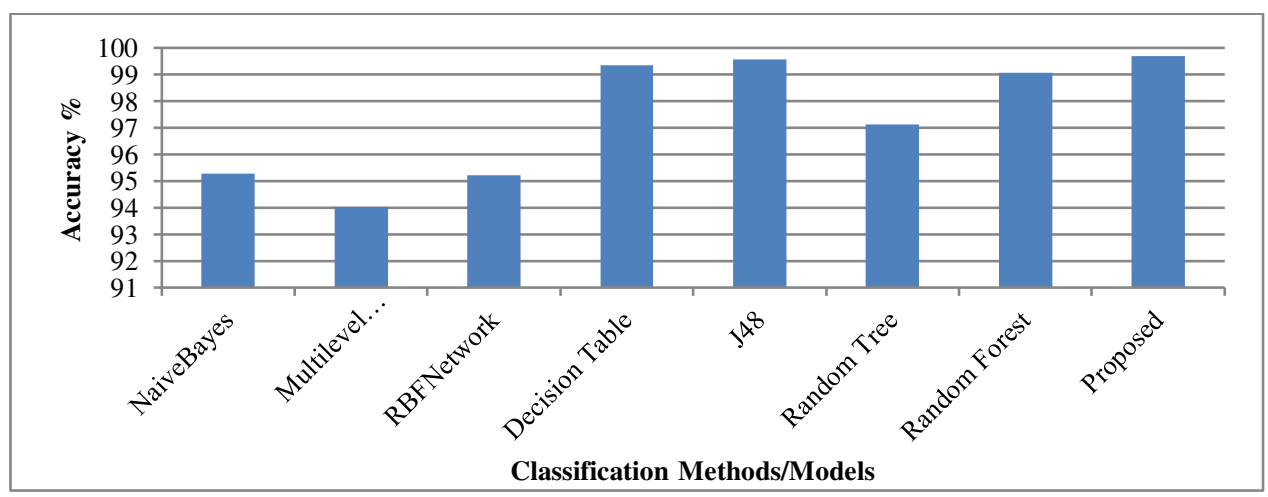

Figure 15: Accuracy Analysis (4 Classes) 
MAE and RMSE are the error based parameters used to analyze the classification error that exist in a model or classifier. The comparative analysis against these parameters is provided in Figure 16. The figure shows that the proposed model achieved the least MAE and RMSE with values .003 and 0.0392. The comparative result shows that the proposed model ensures a significantly lesser error rate than existing classifiers.

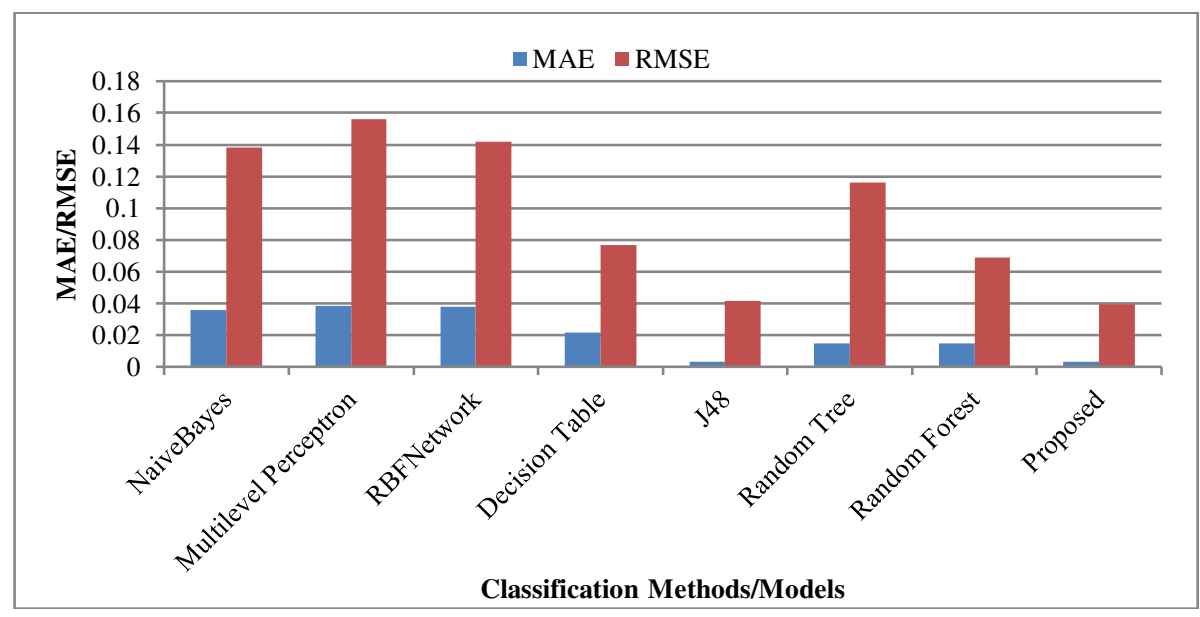

Figure 16: Error Analysis (4 Classes)

The precision and recall parameters analysis are conducted in Figure 17 for validating the relevancy based performance analysis. The Figure shows that the proposed model achieved .996 precision and .997 recall values. The bar graph shows that the results are better than other classifiers and validate the proposed model is more effective, relevant, and reliable for thyroid disease and disease class prediction.

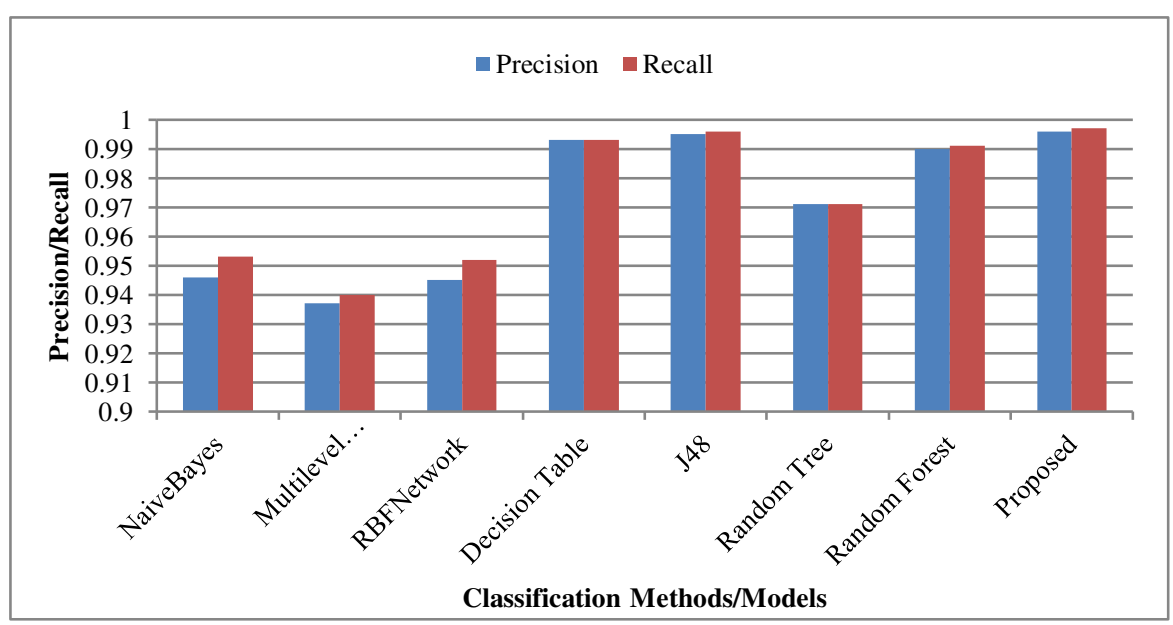

Figure 17: Precision/Recall Analysis (4 Classes) 


\subsection{Comparative Results}

In the previous subsection, the experimentation driven comparative analysis is provided for validating the performance and effectiveness of the proposed model against various classifiers. In this section, the comparative analysis is provided against some other state-of-art methods. The accuracy-based comparative analysis is conducted, and the results are provided in Table 13. The table clearly shows that the proposed model is robust and provided effective results for 2, 4, 6, and 18 class datasets. The maximum accuracy achieved by the proposed model is $99.68 \%$ for predicting the thyroid disease on a 4-classes dataset. The table confirmed that the proposed model outperformed all the existing models and classifiers.

Table 13: Accuracy Analysis against Earlier Studies

\begin{tabular}{|c|c|c|c|c|c|}
\hline & Accuracy & $\begin{array}{l}\text { Number } \\
\text { of } \\
\text { Classes }\end{array}$ & Instances & Attributes & Classes \\
\hline Ahmad1 et al. [28] & 98.5 & 2 & 3163 & 25 & Negative, Hypothyroid \\
\hline Naïve Bayes[34] & 91.63 & 3 & 756 & 22 & $\begin{array}{c}\text { hypothyroidism, } \\
\text { Hyperthyroidism, and } \\
\text { normal. }\end{array}$ \\
\hline Decision Tree[34] & 96.91 & 3 & 756 & 22 & $\begin{array}{c}\text { hypothyroidism, } \\
\text { Hyperthyroidism, and } \\
\text { normal. }\end{array}$ \\
\hline MLP[34] & 95.15 & 3 & 756 & 22 & $\begin{array}{c}\text { hypothyroidism, } \\
\text { hyperthyroidism and } \\
\text { normal. }\end{array}$ \\
\hline RBF Network[34] & 96.03 & 3 & 756 & 22 & $\begin{array}{c}\text { hypothyroidism, } \\
\text { Hyperthyroidism, and } \\
\text { normal. }\end{array}$ \\
\hline $\begin{array}{l}\text { With Filteration } \\
\text { Naïve Bayes[34] }\end{array}$ & 89.96 & 3 & 756 & 19 & $\begin{array}{c}\text { hypothyroidism, } \\
\text { Hyperthyroidism, and } \\
\text { normal. }\end{array}$ \\
\hline $\begin{array}{l}\text { With Filteration } \\
\text { Decision Tree[34] }\end{array}$ & 97.35 & 3 & 756 & 19 & $\begin{array}{c}\text { hypothyroidism, } \\
\text { Hyperthyroidism, and } \\
\text { normal. }\end{array}$ \\
\hline $\begin{array}{l}\text { With Filteration } \\
\text { MLP[34] }\end{array}$ & 94.71 & 3 & 756 & 19 & $\begin{array}{c}\text { hypothyroidism, } \\
\text { Hyperthyroidism, and } \\
\text { normal. }\end{array}$ \\
\hline $\begin{array}{l}\text { With Filteration RBF } \\
\text { Network[34] }\end{array}$ & 94.27 & 3 & 756 & 19 & $\begin{array}{c}\text { hypothyroidism, } \\
\text { Hyperthyroidism, and } \\
\text { normal. }\end{array}$ \\
\hline Shankar et al.[35] & 97.49 & 3 & 7547 & 30 & $\begin{array}{l}\text { Hypothyroid, Hyper } \\
\text { Thyroid, Normal }\end{array}$ \\
\hline $\mathrm{KNN}[36]$ & 93.44 & 3 & 7200 & 21 & $\begin{array}{c}\text { Hypothyroidism, } \\
\text { Hyperthyroidism, Normal }\end{array}$ \\
\hline Naïve Bayes[36] & 22.56 & 3 & 7200 & 21 & Hypothyroidism, \\
\hline
\end{tabular}




\begin{tabular}{|c|c|c|c|c|c|}
\hline $\begin{array}{c}\text { Proposed (Level 2 } \\
\text { Filter) }\end{array}$ & 98.877 & 2 & 9172 & 21 & Hyperthyroidism, Normal \\
\hline $\begin{array}{c}\text { Proposed (Level 2 } \\
\text { Filter) }\end{array}$ & 97.6014 & 6 & 9172 & 21 & $\begin{array}{c}\text { Positive, Negative } \\
\text { Binding_Protein, } \\
\text { Replacement_Theory, } \\
\text { General_Health, } \\
\text { Antithyroid_treatment }\end{array}$ \\
\hline $\begin{array}{c}\text { Proposed (Level 2 } \\
\text { Filter) }\end{array}$ & 96.2495 & 18 & 3772 & 21 & $\begin{array}{c}\text { NEG,F,A,I,M,N,G,K,J,L,Q, } \\
\text { C,O,H,D,P,B,E }\end{array}$ \\
\hline $\begin{array}{c}\text { Proposed (Level 2 } \\
\text { Filter) }\end{array}$ & 99.3637 & 2 & 3772 & 21 & $\begin{array}{c}\text { Positive, Negative } \\
\text { Proposed (Level 2 } \\
\text { Filter) }\end{array}$ \\
\hline
\end{tabular}

\section{Conclusion}

In this paper, a relevant and reliable feature processed ELM classifier is proposed for improving the performance for prediction of thyroid existence and type. The proposed framework included a wide pre-processing stage called Filtered-ExpandedFiltered for transiting the raw-dataset to relevant-featureset. The feature filtration in this framework is performed at two levels. At level-I, the raw dataset is processed, and a fuzzy-based composite-measure is applied along with an expert recommendation for identifying the most contributing ranked features. In this state, the composite-fuzzy rules are generated on InfoGain, GainRatio, and ChiSq measures. This filtered dataset is further processed by the statistical-distance based measure for generating the larger and aggregative analysis based featureset. At the second filtration stage, the fuzzy rule is applied to this expanded dataset for transiting the continuous values to fuzzycategorical values. The fuzzy-based composite measure is again applied at this level, and a relevant and effective dataset is obtained. This filtered dataset is finally processed by a dynamic weight adjustment based ELM classifier for accurate prediction of thyroid disease existence $d$ type. The proposed model experiments on two datasets for five trials. The number of classes in these trails is 2,4,6, and 18. The analytical observations are conducted against error based, accuracy, and relevancy based parameters. The experimentation based comparative analysis is conducted against NaiveBayes, MultiLevel Perceptron, RBF Network, Decision tree, Decision Table, Random Tree, and Random Forest classifiers. The results confirmed that the proposed framework achieved the better accuracy, Precision and Recall rate and reduced the RMSE and MAE. The proposed framework also achieved better accuracy against other state-of-art methods and models. In the future, the bioinformatics features can be used for enhancing the capabilities, performance, and effectiveness of 
the proposed model. The proposed model can be tested on other diseases by collecting the symptoms, behavior, and features of that domain.

\section{DECLARATIONS}

\section{FUNDING}

No funds received for this research.

\section{CONFLICTS OF INTEREST}

There is no conflict of interest, financial or others. I as sole author ensured the ethics approval and participation of the research.

\section{AVAILABILITY OF DATA AND MATERIAL}

The datasets described in the paper are cited properly.

\section{CODE AVAILABILITY}

It is part of ongoing and extendable research. Code sharing is not possible. 


\section{References}

[1] B. Xu and R. Ghossein, "Evolution of the histologic classification of thyroid neoplasms and its impact on clinical management," European Journal of Surgical Oncology (EJSO), pp. 1-10, 2017.

[2] Q. Pan, Y. Zhang, M. Zuo, L. Xiang, and D. Chen, "Improved Ensemble Classification Method of Thyroid Disease Based on Random Forest," in 8th International Conference on Information Technology in Medicine and Education (ITME), Fuzhou, 2016, pp. 567-571.

[3] I. M. D. Maysanjaya, H. A. Nugroho, and N. A. Setiawan, "A comparison of classification methods on diagnosis of thyroid diseases," in International Seminar on Intelligent Technology and Its Applications (ISITIA), Surabaya, 2015, pp. 89-92.

[4] C. Y. Chang, M. F. Tsai, and S. J. Chen, "Classification of the thyroid nodules using support vector machines," in IEEE International Joint Conference on Neural Networks (IEEE World Congress on Computational Intelligence), Hong Kong, 2008, pp. 3093-3098.

[5] M. A. Qureshi and K. Eksioglu, "Expert advice ensemble for thyroid disease diagnosis," in 25th Signal Processing and Communications Applications Conference (SIU), Antalya, 2017, pp. 1-4.

[6] Priyanka Duggal and Shipra Shukla, "Prediction Of Thyroid Disorders Using Advanced Machine Learning Techniques," 10th International Conference on Cloud Computing; Data Science \& Engineering, pp. 670-675, 2020.

[7] Shaik Razia and M.R.Narasingarao, "A Neuro Computing Framework for Thyroid Disease Diagnosis using Machine Learning Techniques," Journal of Theoretical and Applied Information Technology, vol. 95, no. 9, pp. 1996-2005, 2017.

[8] Shaik Razia and M. R. Narasinga Rao, "Machine Learning Techniques for Thyroid Disease Diagnosis - A Review," Indian Journal of Science and Technology, vol. 9, no. 28, pp. 1-9, 2016.

[9] Shaik Razia, M.R.Narasingarao, and Polaiah Bojja, "Development and Analysis of Support Vector Machine Techniques for Early Prediction of Breast Cancer and Thyroid," Journal of Advanced Research in Dynamical and Control Systems, vol. 9, no. 6, pp. 869878, 2017.

[10] Irina IoniŃă and Liviu Ionita, "Prediction of Thyroid Disease Using Data Mining Techniques," BRAIN. Broad Research in Artificial Intelligence and Neuroscience, vol. 7, no. 3, pp. 115-124, 2016.

[11] Khushboo Chandel, Veenita Kunwar, Sai Sabitha, Tanupriya Choudhury, and Saurabh Mukherjee, "A comparative study on thyroid disease detection using K-nearest neighbor and Naive Bayes classification techniques," CSI Transactions on ICT, pp. 1-7, 2017.

[12] Shaik Razia, M.R. Narasingarao, and G.R. Sridhar, "A Decision Support System for Prediction of Thyroid Disease- A Comparison of Multilayer Perceptron Neural Network and Radial Basis Function Neural Network," Journal of Theoretical and Applied Information Technology, vol. 80, no. 3, pp. 544-551, 2015.

[13] Umar Sidiq and Syed Mutahar Aaqib, "An Empirical Model for Thyroid Disease Diagnosis Using Data Mining Techniques," International Conference on Sustainable Communication Networks and Application, pp. 589-597, 2019.

[14] Sagar Raisinghani, Rahul Shamdasani, Mahima Motwani, Amit Bahreja, and Priya Raghavan Nair Lalitha, "Thyroid Prediction Using Machine Learning Techniques," International Conference on Advances in Computing and Data Sciences, pp. 140-150, 2019. 
[15] Amina Begum and A Parkavi, "Prediction of thyroid Disease Using Data Mining Techniques," 5th International Conference on Advanced Computing \& Communication Systems, pp. 342-345, 2019.

[16] D.Hemalatha and S. Poorani, "Supervised Machine Learning Models For Classification Of Thyroid Data," International Journal of Scientific and Technology Research, vol. 9, no. 2, pp. 1683-1685, 2020.

[17] Yasir Iqbal and Sonu Mittal, "Thyroid Disease Prediction Using Hybrid Machine Learning Techniques: An Effective Framework," International Journal of Scientific and Technology Research, vol. 9, no. 2, pp. 2868-2874, 2020.

[18] S. Shroff, S. Pise, P. Chalekar, and S. S. Panicker, "Thyroid disease diagnosis: A survey," in IEEE 9th International Conference on Intelligent Systems and Control (ISCO), Coimbatore, 2015, pp. 1-6.

[19] Zhuang Li, Jingyan Qin, Xiaotong Zhang, and Yadong Wan, "A Hybrid Intelligent Framework for Thyroid Diagnosis," International Conference on Cyberspace Data and Intelligence, pp. 441-451, 2019.

[20] Arvind Selwal and Ifrah Raoof, "A multi-layer perceptron based improved thyroid disease prediction system," Indonesian Journal of Electrical Engineering and Computer Science, vol. 17, no. 1, pp. 524-533, 2019.

[21] Dhyan Chandra Yadav and Saurabh Pal, "Decision Tree Ensemble Techniques To Predict Thyroid Disease," International Journal of Recent Technology and Engineering (IJRTE), vol. 8, no. 3, pp. 8242-8246, 2019.

[22] Bhavna Dharamkar, Praneet Saurabh, Ritu Prasad, and Pradeep Mewada, "An Ensemble Approach for Classification of Thyroid Using Machine Learning," Progress in Computing; Analytics and Networking, pp. 13-22, 2020.

[23] S. Dash, M. N. Das, and B. K. Mishra, "Implementation of an optimized classification model for prediction of hypothyroid disease risks," in International Conference on Inventive Computation Technologies (ICICT), Coimbatore, 2016, pp. 1-4.

[24] S. A. Biyouki, I. B. Turksen, and M. H. F. Zarandi, "Fuzzy rule-based expert system for diagnosis of thyroid disease," in IEEE Conference on Computational Intelligence in Bioinformatics and Computational Biology (CIBCB), Niagara Falls, 2015, pp. 1-7.

[25] Li Huang et al., "Comparing of feature selection and classification methods on reportbased subhealth data," in IEEE International Conference on Bioinformatics and Biomedicine (BIBM), Shenzhen, 2016, pp. 1356-1358.

[26] J. M. Rodriguez, D. Godoy, and A. Zunino, "An Empirical Comparison Of Feature Selection Methods In Problem Transformation Multi-label Classification," in IEEE Latin America Transactions, vol. 14 , 2016, pp. 3784-3791.

[27] D Lakshmi Padmaja and B Vishnuvardhan, "Comparative Study of Feature Subset Selection Methods for Dimensionality Reduction on Scientific Data," in 6th International Advanced Computing Conference, 2016, pp. 31-34.

[28] V. Prasad, T. Srinivasa Rao, and M. Surendra Prasad Babu, "Thyroid disease diagnosis via hybrid architecture composing rough data sets theory and machine learning algorithms," Soft Computing, pp. 1179-1189, 2016.

[29] Wei-Chang Yeh, "Novel swarm optimization for mining classification rules on thyroid gland data," Information Sciences, vol. 197, pp. 65-76, 2012.

[30] Esin Dogantekin, Akif Dogantekin, and Derya Avci, "An expert system based on Generalized Discriminant Analysis and Wavelet Support Vector Machine for diagnosis of thyroid diseases," Expert Systems with Applications, vol. 38, no. 1, pp. 146-150, 2011. 
[31] Yoichi Hayashi, Satoshi Nakano, and Shota Fujisawa, "Use of the recursive-rule extraction algorithm with continuous attributes to improve diagnostic accuracy in thyroid disease," Informatics in Medicine Unlocked, pp. 1-8, 2015.

[32] Feyzullah Temurtas, "A comparative study on thyroid disease diagnosis using neural networks," Expert Systems with Applications, vol. 36, no. 1, pp. 944-949, 2009.

[33] Halife Kodaz, Seral Ozsen, Ahmet Arslan, and Salih Gunes, "Medical application of information gain based artificial immune recognition system (AIRS): Diagnosis of thyroid disease," Expert Systems with Applications, vol. 36, no. 2 Part 2, pp. 3086-3092, 2009.

[34] Kemal Polat, Seral Sahan, and Salih Gunes, "A novel hybrid method based on artificial immune recognition system (AIRS) with fuzzy weighted pre-processing for thyroid disease diagnosis," Expert Systems with Applications, vol. 32, no. 4, pp. 1141-1147, 2007.

[35] Wei-Wen Chang, Wei-Chang Yeh, and Pei-Chiao Huang, "A hybrid immune-estimation distribution of algorithm for mining thyroid gland data," Expert Systems with Applications, vol. 37, no. 3, pp. 2066-2071, 2010.

[36] Waheed Ahmad, Ayaz Ahmad, Chuncheng Lu, Barkat Ali Khoso, and Lican Huang, "A novel hybrid decision support system for thyroid disease forecasting," Soft Computing, pp. $1-7,2018$.

[37] Rekha Pal, Tanvi Anand, and Sanjay Kumar Dubey, "Evaluation and performance analysis of classification techniques for thyroid detection," International Journal Business Information System, vol. 28, no. 2, pp. 163-177, 2018.

[38] K. Shankar, S. K. Lakshmanaprabu, Deepak Gupta, Andino Maseleno, and Victor Hugo C. de Albuquerque, "Optimal feature-based multi-kernel SVM approach for thyroid disease classification," The Journal of Supercomputing, vol. 76, pp. 1128-1143, 2020.

[39] http://repository.seasr.org. [Online]. http://repository.seasr.org/Datasets/UCI/arff/

[40] https://www.healthline.com. [Online]. https://www.healthline.com/health/t4-test

[41] https://www.thyroid.org. [Online]. https://www.thyroid.org/thyroid-information/

[42] https://sourceforge.net.

https://sourceforge.net/projects/meka/files/Datasets/thyroid-L7.arff/

download?use mirror=excellmedia\&download=\&failedmirror $=$ kent .dl.sourceforge.net 
Figures

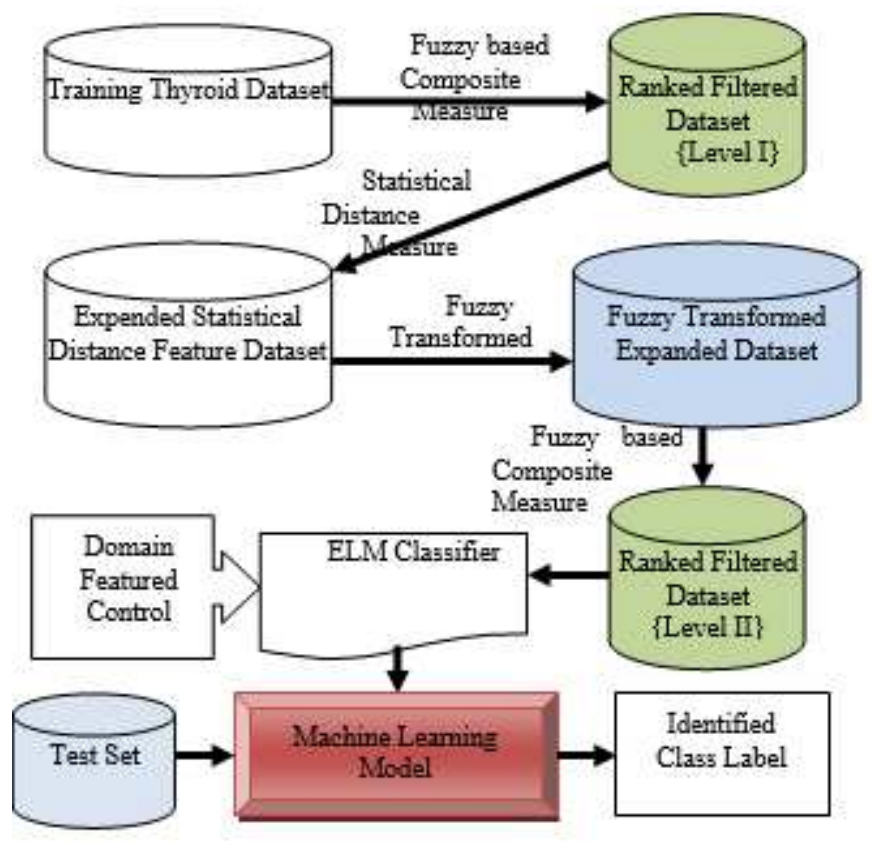

Figure 1

Fuzzy Filtered-Expended-Filtered Featured based ELM Model

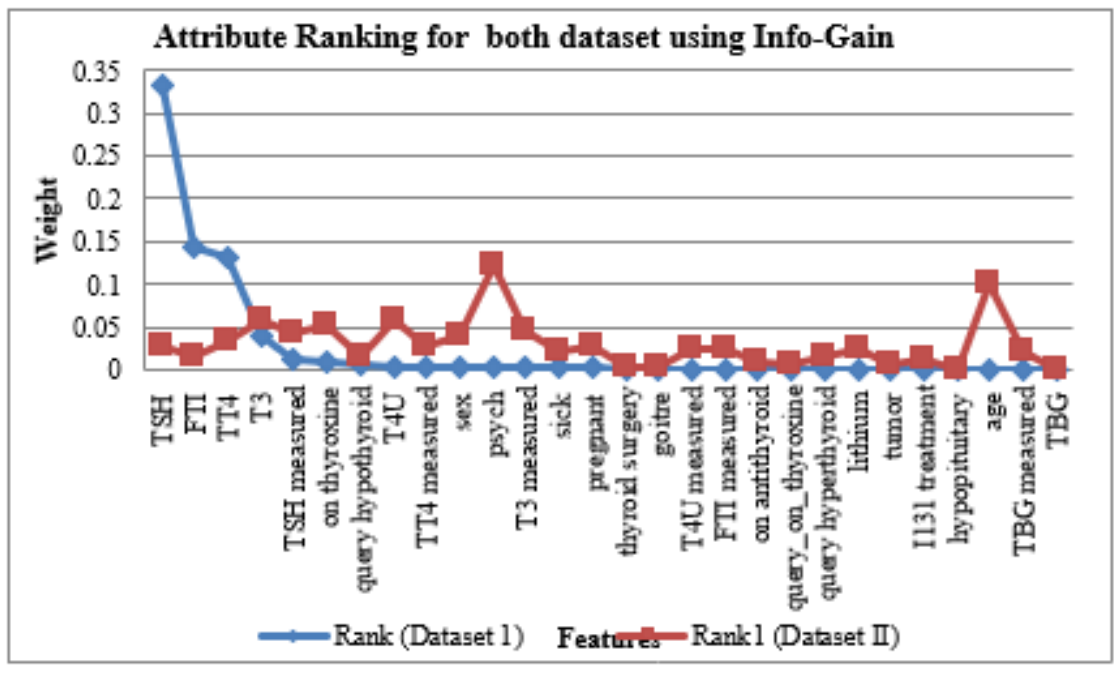

Figure 2

Attribute Ranking for both Datasets 


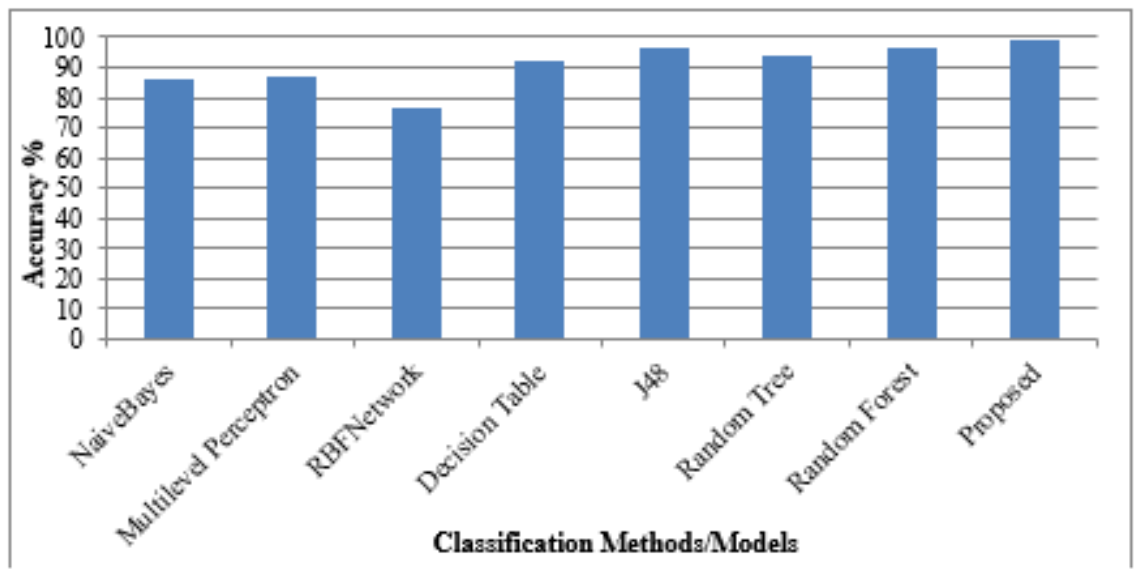

Figure 3

Accuracy analysis (2-Classes)

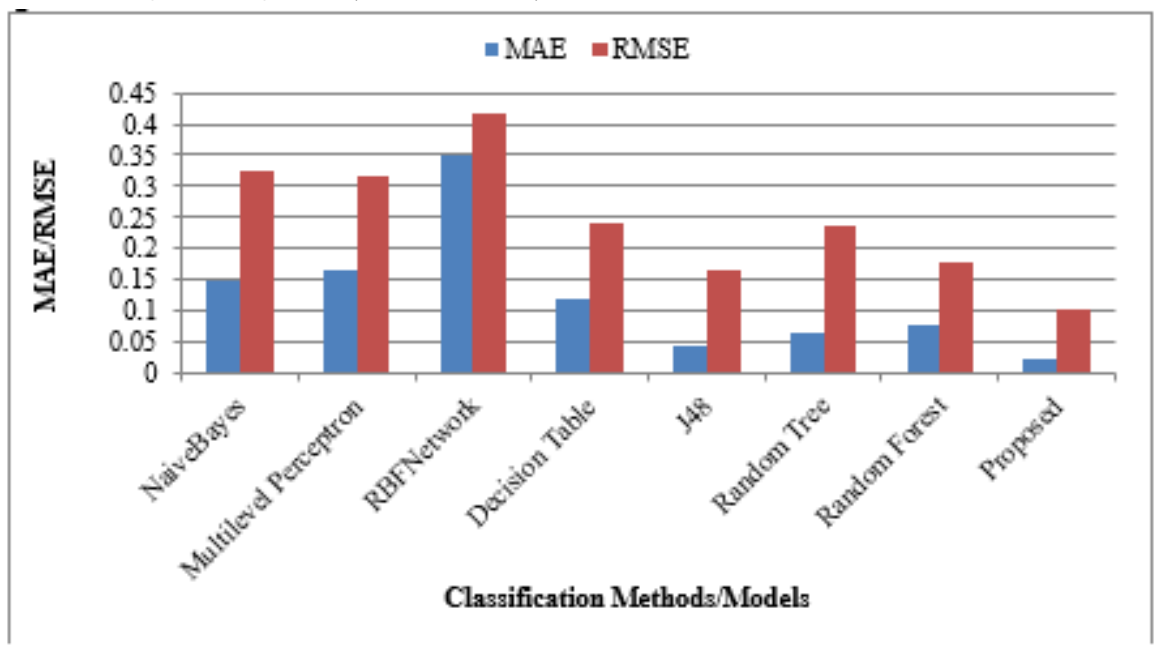

Figure 4

Error Analysis (2-Classes)

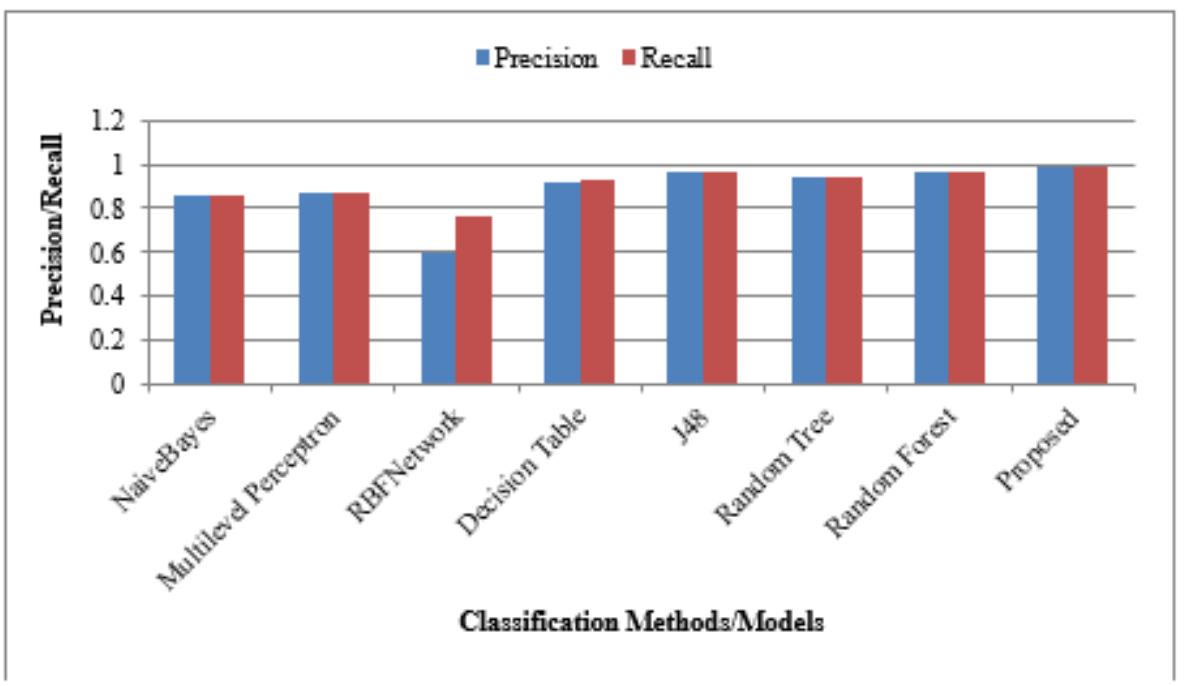

Figure 5 
Precision/Recall Analysis (2-Classes)

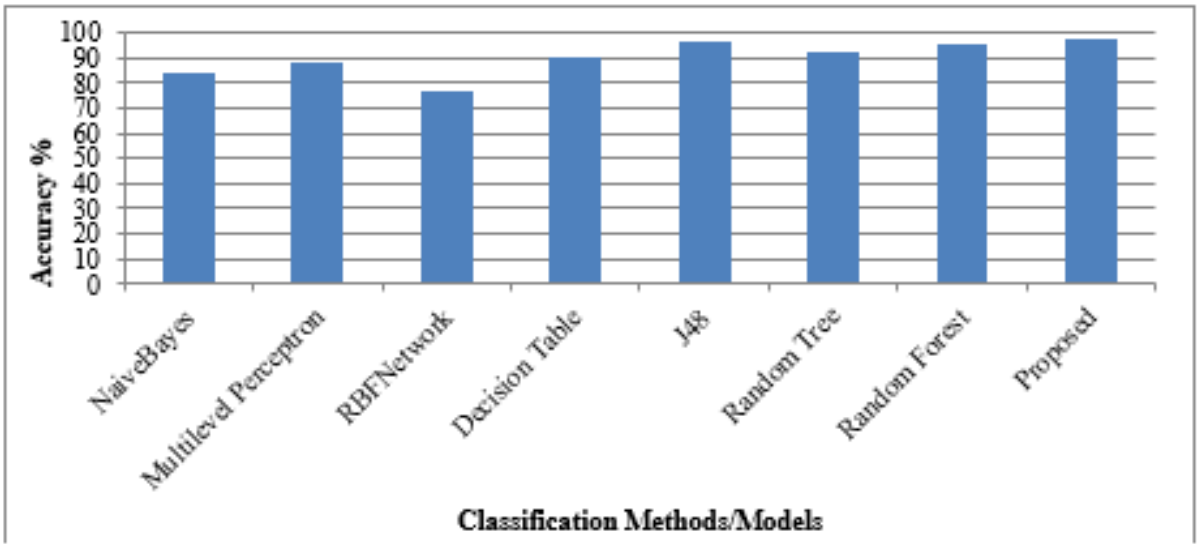

Figure 6

Accuracy analysis on Disease Class (6-Classes)

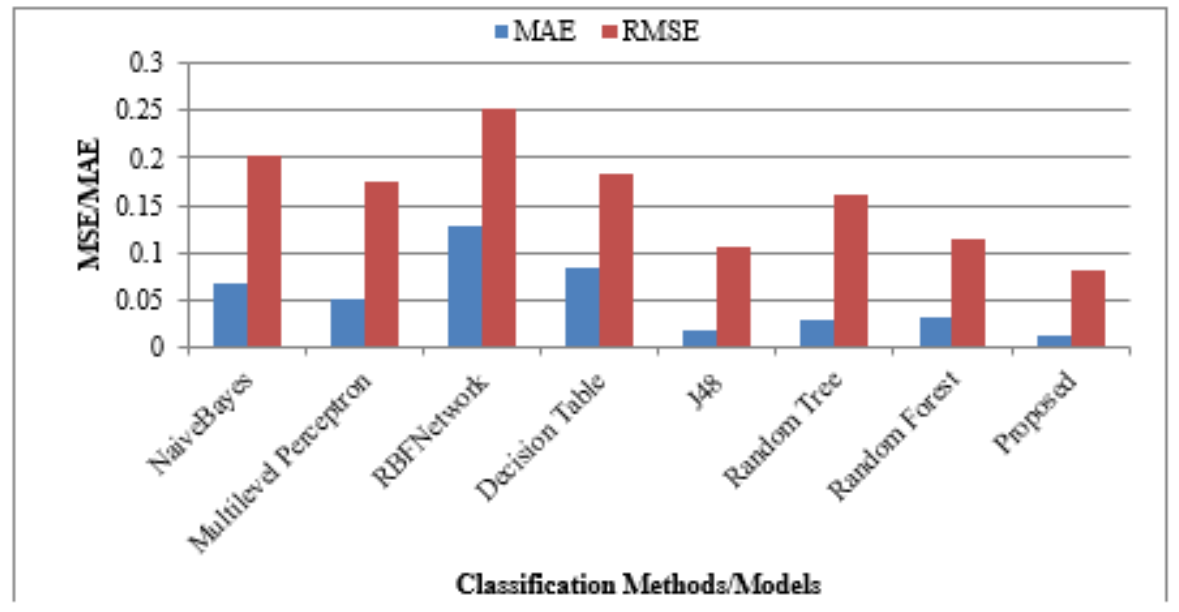

Figure 7

Error analysis on Disease Class (6-Classes)

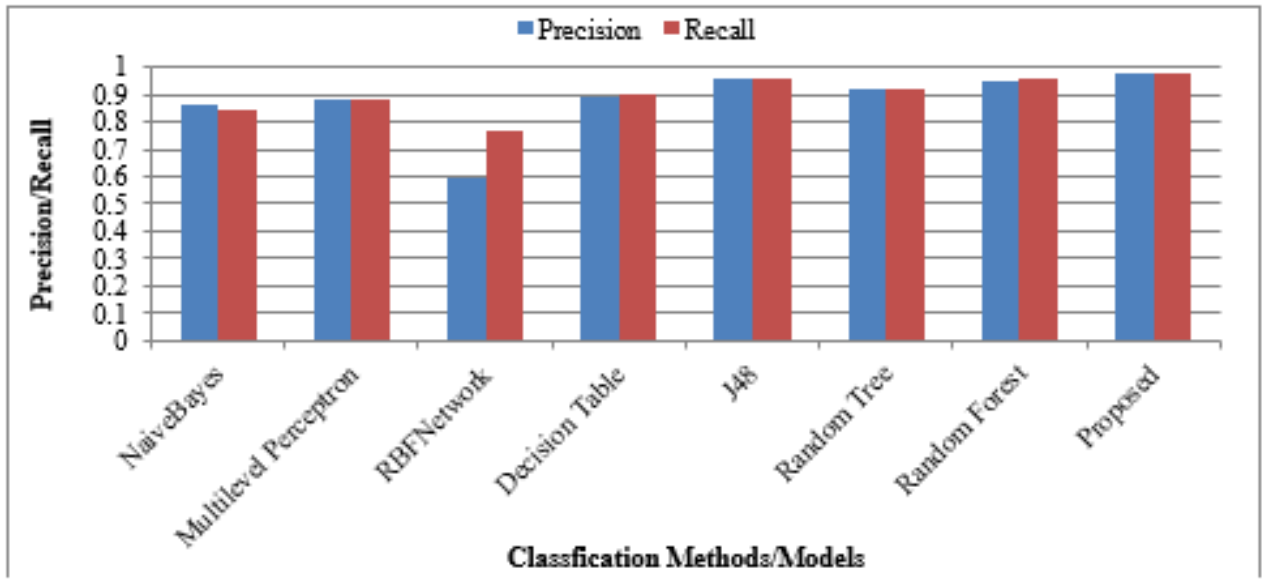

Figure 8 
Precision and Recall Analysis (6-Classes)

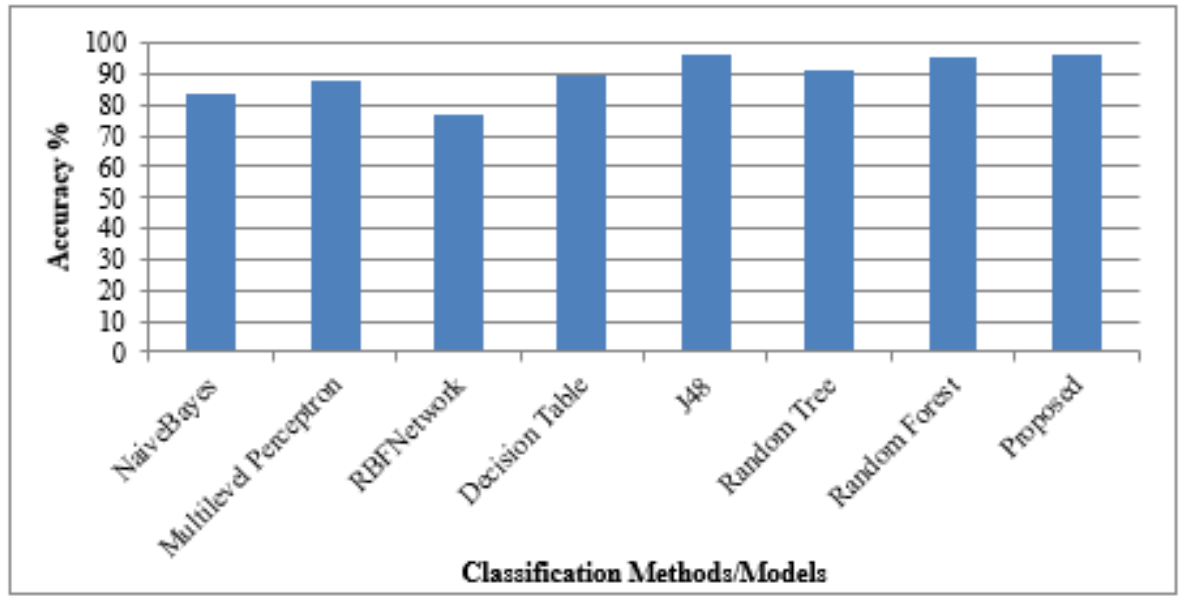

Figure 9

Accuracy Analysis (18 Classes)

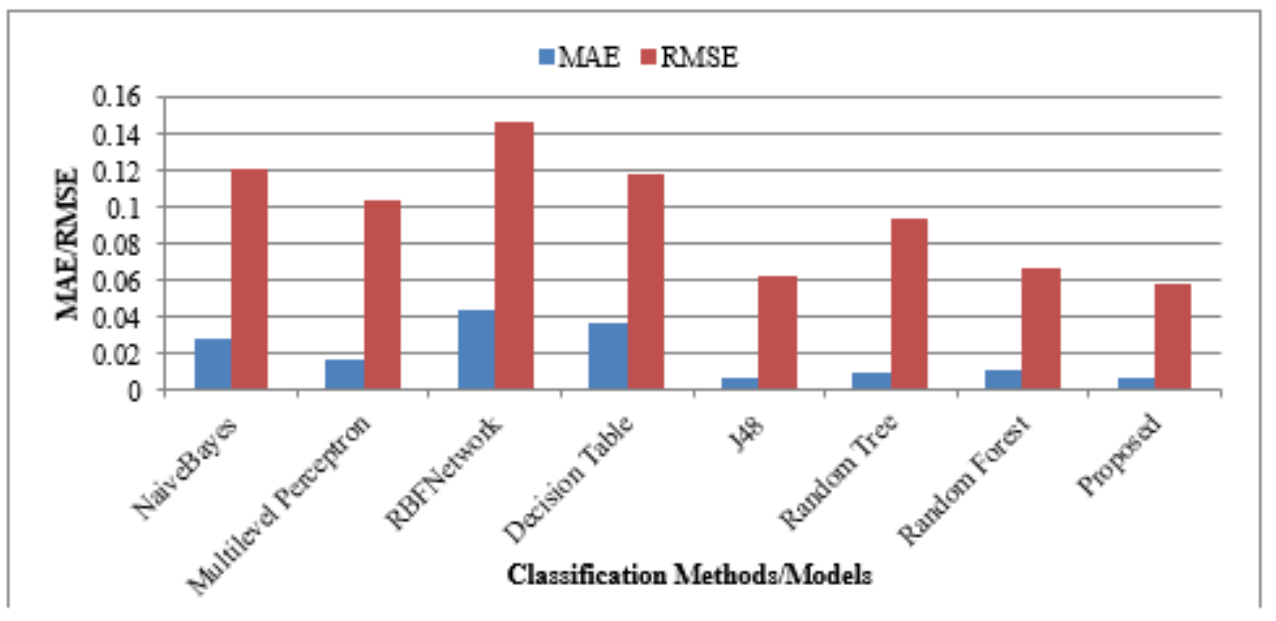

Figure 10

Error Analysis (18 Classes)

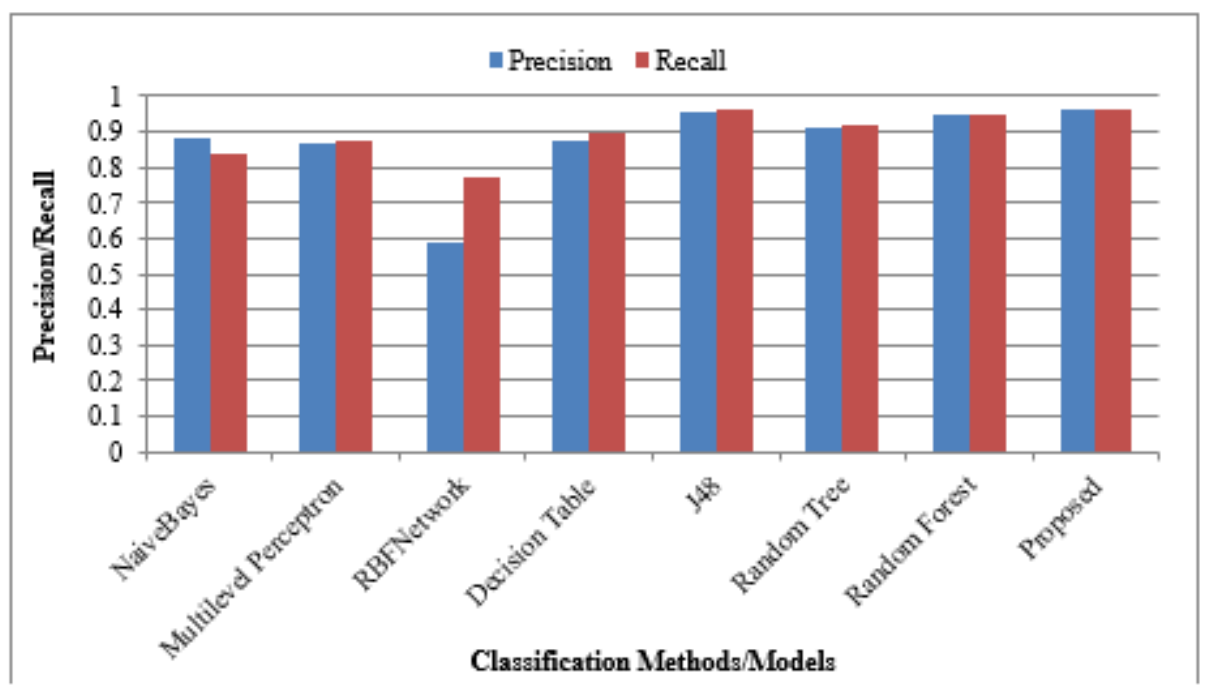


Figure 11

Precision/Recall Analysis (18 Classes)

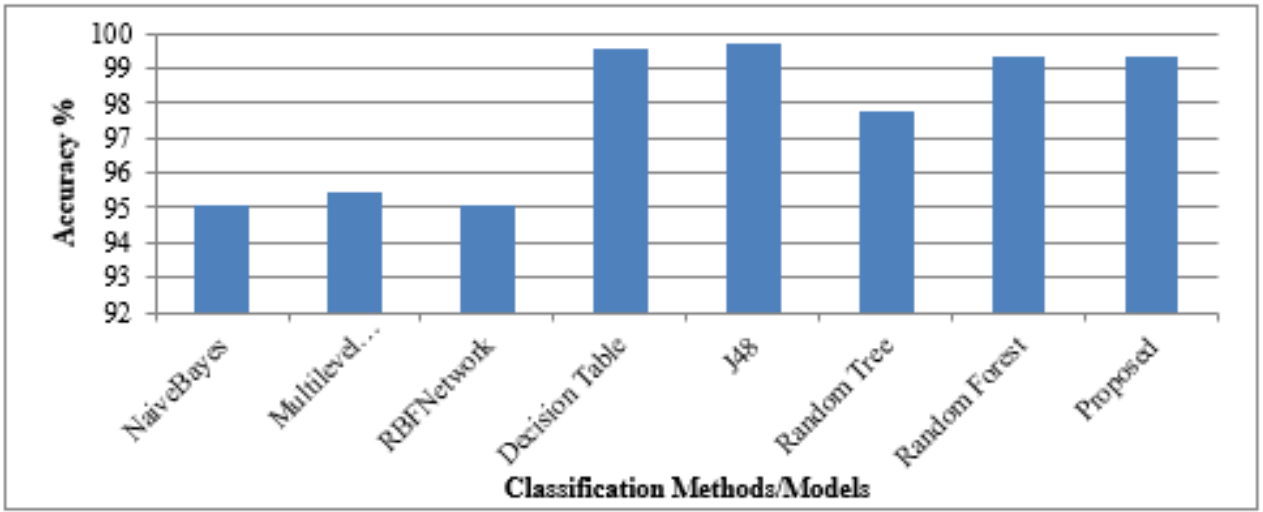

Figure 12

Accuracy Analysis (2 Classes)

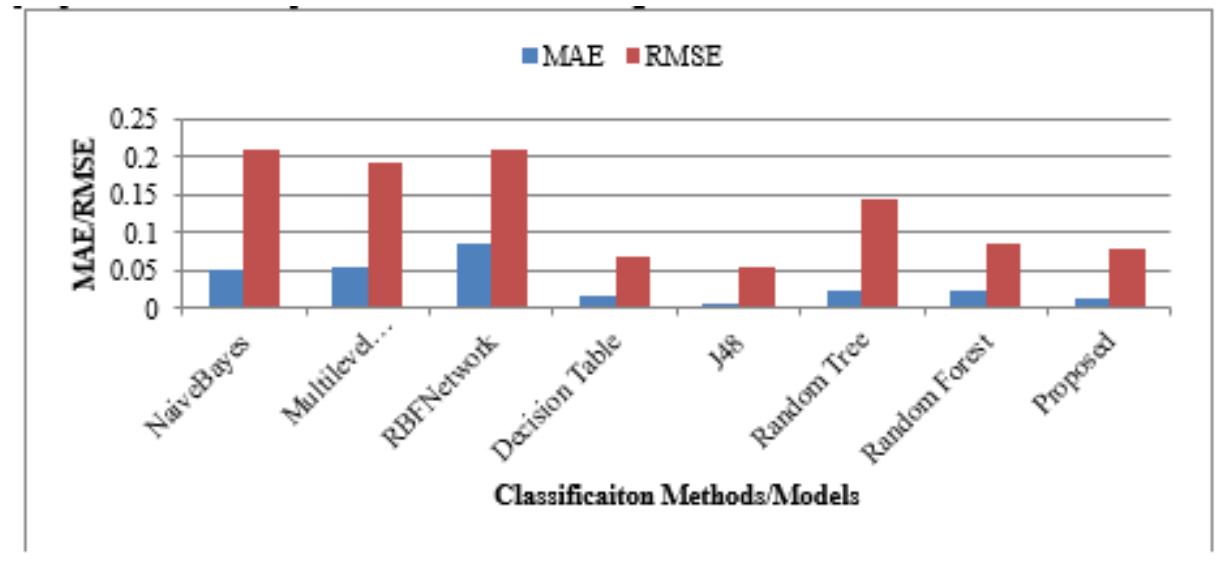

Figure 13

Error Analysis (2 Classes)

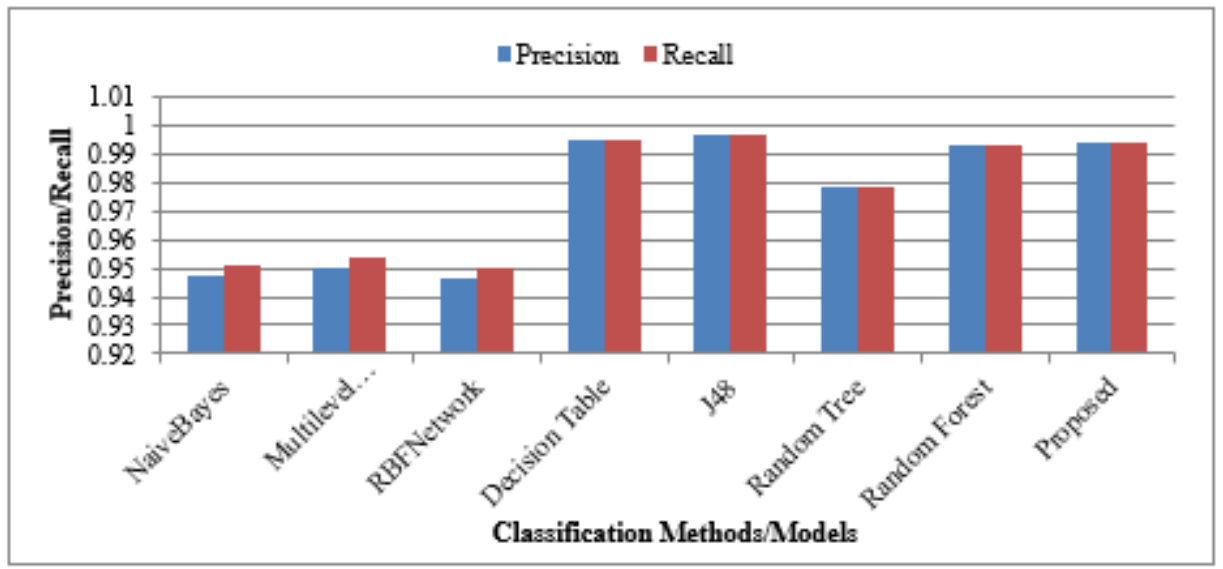

Figure 14 
Precision/Recall Analysis

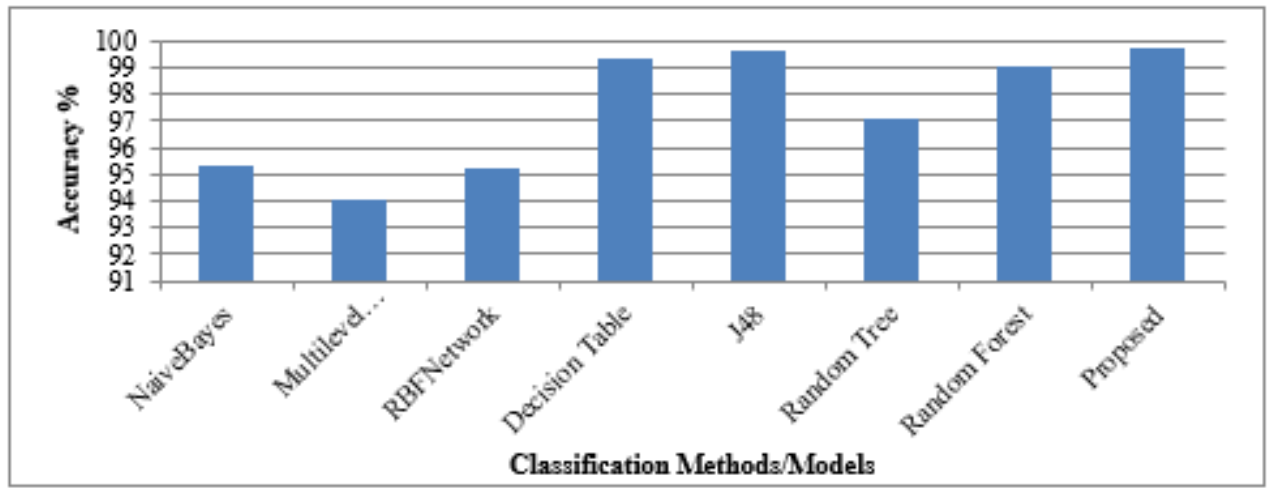

Figure 15

Accuracy Analysis (4 Classes)

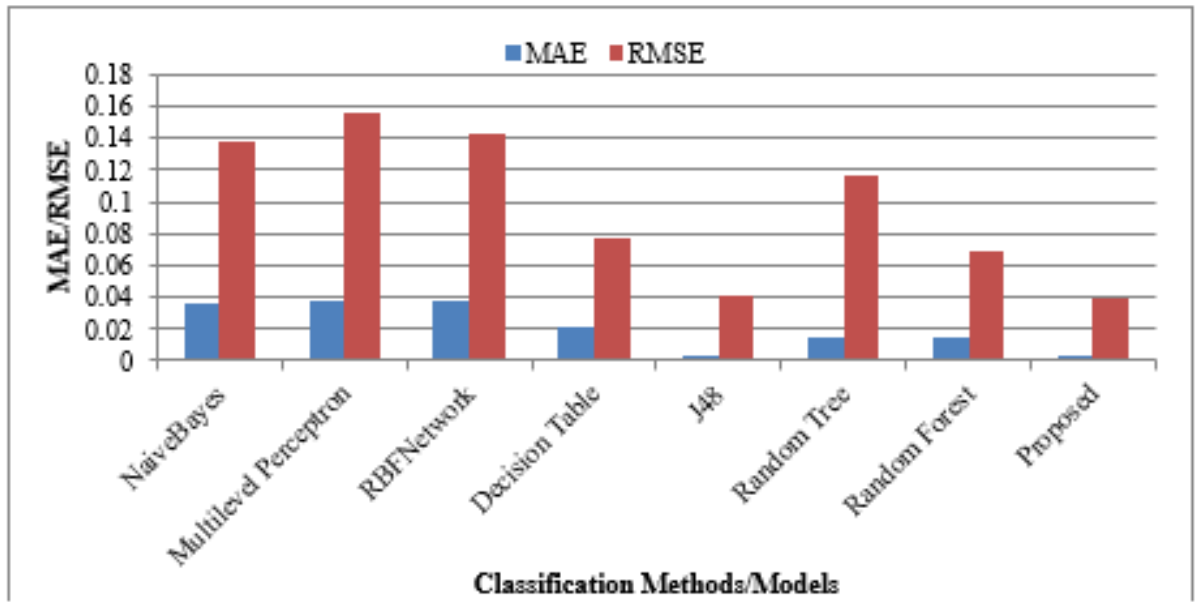

Figure 16

Error Analysis (4 Classes)

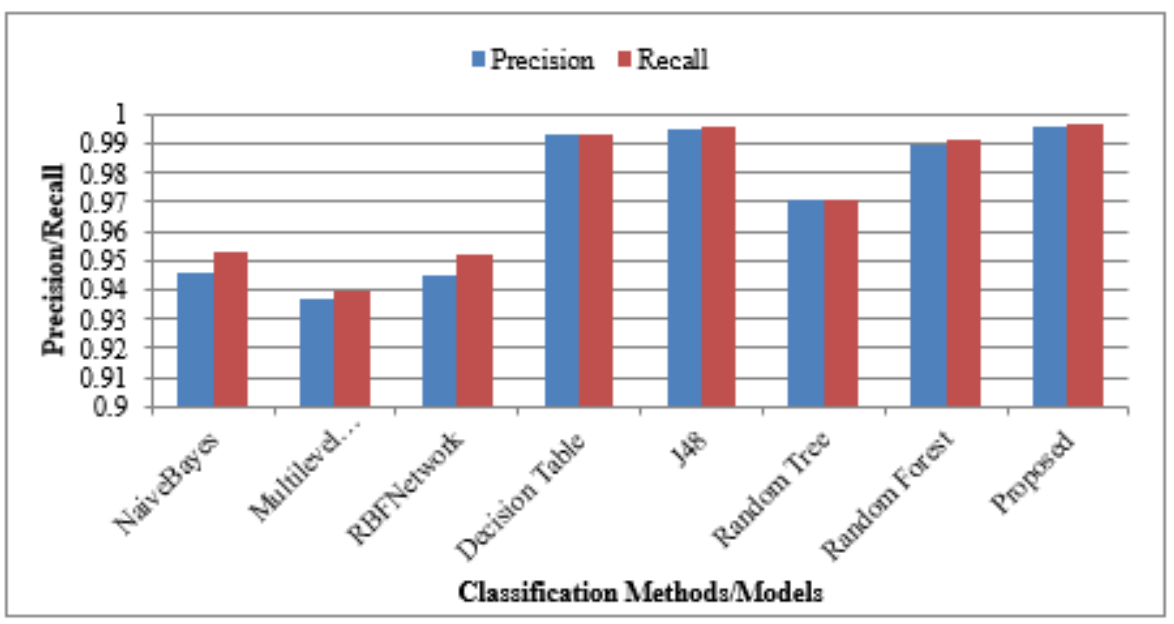

Figure 17

Precision/Recall Analysis (4 Classes) 